\title{
Sporadic wind wave horse-shoe patterns
}

\author{
S. Yu. Annenkov ${ }^{1}$ and V. I. Shrira ${ }^{2}$

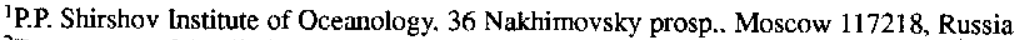 \\ 2Department of Applied Mathematics. University College Cork, Cork, Ireland
}

Received: 01 December 1998 - Accepted: 19 February 1999

\begin{abstract}
The work considers three-dimensional crescent-shaped patterns often seen on water surface in natural basins and observed in wave tank experiments. The most common of these 'horse-shoe-like' patterns appear to be sporadic, i.e., emerging and disappearing spontaneously even under steady wind conditions. The paper suggests a qualitative model of these structures aimed at explaining their sporadic nature, physical mechanisms of their selection and their specific asymmetric form.
\end{abstract}

First, the phenomenon of sporadic horse-shoe patterns is studied numerically using the novel algorithm of water waves simulation recently developed by the authors (Annenkov and Shrira, 1999). The simulations show that a steep gravity wave embedded into widespectrum primordial noise and subjected to small nonconservative effects typically follows the simple evolution scenario: most of the time the system can be considered as consisting of a basic wave and a single pair of oblique satellites, although the choice of this pair tends to be different at different instants. Despite the effective low-dimensionality of the multimodal system dynamics at relatively short time spans, the role of small satellites is important: in particular, they enlarge the maxima of the developed satellites. The presence of Benjamin-Feir satellites appears to be of no qualitative importance at the timescales under consideration.

The selection mechanism has been linked to the quartic resonant interactions among the oblique satellites lying in the domain of five-wave (McLean's class II) instability of the basic wave: the satellites tend to push each other out of the resonance zone due to the frequency shifts caused by the quartic interactions. Since the instability domain is narrow (of order of cube of the basic wave steepness), eventually in a generic situation only a single pair survives and attains considerable amplitude. The specific front asymmetry is found to result from the interplay of quartic and quintet interactions and non-

Correspondence to: $\mathrm{V}$. I. Shrira conservative effects: the growing and grown satellites have a specific value of phase with respect to the basic wave that corresponds to downwind orientation of the convex sides of wave fronts. As soon as the phase relation is violated, the satellite's amplitude quickly decreases down to the noise level.

\section{Introduction}

Wind waves in natural basins display a variety of threedimensional patterns. Among them, patterns of spectacular crescent-shaped form, resembling horse-shoes, can be often discerned. The challenge to understand and describe mathematically these patterns, apart from the natural desire to explain a commonly observed phenomenon, comes from two different sides. On the one hand, the patterns are very important from the ocean science perspective, since they modify the airflow above the surface and thus affect the air-sea momentum transfer. They also change in a specific way the radar scattering from the sea surface (Shrira et al, 1999), and, last but not least, they require conceptually new models to describe statistically wind-wave field dynamics in their presence (Shrira et al, 1996). On the other hand, the phenomenon is of true interest from the viewpoint of nonlinear science, as a new non-trivial scenario of pattern formation having no close analogues in the very rich literature on patterns (e.g. Bowman and Newell, 1998).

Though no field studies of these horse-shoe patterns are known, their main features inferred from common observations were briefly summarized in Shrira et al (1996) (below referred to as SBK). Among these features are:

(i) wave fronts are of crescent-shaped form and are always oriented forward, i.e., their convex sides look downwind; 
(ii) the patterns appear quickly after the onset of a fresh wind, at early stages of wave development characterized by high wave steepness;

(iii) the patterns might be localized in space and time, but exist over space- and timescales greatly exceeding the basic wave wavelength and period.

A number of experiments carried out in wave tanks (Su et al, 1982; Collard and Caulliez, 1999) and windwave facilities (Melville, 1982; Kusaba and Mitsuyasu, 1986; Collard and Caulliez, 1999), both in the presence and absence of wind, made it possible to reproduce the patterns in their ideal form under controlled conditions and to measure their parameters. It has been established (first suggested by Su et al, 1982; Melville, 1982 and later confirmed by Collard and Caulliez, 1999) that the inception mechanism of the horse-shoes is indeed due to McLean's class II instability (five-wave decay) of a plane basic wave. It was also found that wave steepness above a certain threshold is necessary for their inception. However, specific mechanisms of the pattern formation remain unidentified.

SBK, having summarized the available observations and a number of theoretical works aimed at explaining the phenomenon qualitatively, suggested a possible scenario of the emergence of the patterns. The authors made the assumption that the single symmetric pair with the largest linear growth rate due to McLean's class II instability prevails, while all other pairs (there is a continuum of them lying within the instability domain drawn by McLean (McLean, 1982; Craik, 1985)) may be ignored. Although such an approach is common in studies of hydrodynamic stability and is plausible in some cases (e.g. Craik, 1985), in general, it does require justification for each particular case. Relying mainly upon the experimental evidence and giving some arguments of qualitative character in support of this hypothesis, SBK confined themselves to the consideration of a lowdimensional system composed of a basic wave and the fastest growing pair of satellites. The resulting patterns indeed render the specific geometry closely resembling the observed horse-shoe forms, but only under a special relation between the phases of the interacting waves. Namely, the 'effective phase' $\theta=3 \alpha-2 \beta$, where $\alpha$ and $\beta$ are the phases of complex amplitude of the envelope of the basic wave and symmetric satellites respectively, must be negative, the best agreement with observations corresponding to the case $\theta=-\pi / 2$. Any credible theoretical model aimed at describing the phenomenon of horse-shoe patterns first should be able to explain this specific asymmetry. It should be noted that in a more general context the seemingly similar crescent-shaped patterns often emerge in different hydrodynamic situations, but the underlying mechanisms are quite distinct. For example, the migrating barchan dunes having well pronounced crescent shape are always oriented backward, i.e., their convex sides look upwind.
To address the specific front asymmetry, SBK first analytically considered the class II instability of a basic wave, applying the Zakharov equation to this simple three-wave problem known to be integrable (Shemer and Stiassnie, 1985). Within this framework, no preference for phases was found. Instead, the phase of a satellite during the cycle of its growth and subsequent decay rotates through all the possible values, giving a full range of various three-dimensional patterns of all orientations with overall distribution being symmetric in upand downwind directions, in the apparent contradiction to the observations. However, SBK showed that nonconservative effccts, inserted into the system but small enough to preserve the Hamiltonian structure to the required order, would create in the phase space absolutely attractive foci, the patterns corresponding to them bearing all the necessary features of those observed.

In this sense, SBK have built a plausible qualitative theory of the emergence of long-lived crescent-shaped patterns. The presence of non-conservative effects allows the simplest three-wave system to evolve into a steady state with the geometric form of the free surface with the required characteristics. Still, a number of important questions were left unanswered or discussed at a qualitative level only.

The most crude assumption was the selection of a single symmetric pair of oblique satellites for the model of the class II instability. Although the domain of the fivewave instability is $O\left(\varepsilon^{3}\right)$ narrow, $\varepsilon$ being the measure of the basic wave steepness, it is still continuous and there is no a priori reason to confine the consideration of this instability to a single pair of satellite harmonics even if this pair is characterized by the maximal linear growth rate. Moreover, this approach completely neglects the sideband satellites growing due to the Benjamin-Feir instability. Besides that, despite the recent advances in understanding of global dynamics within the framework of the truncated three-mode system (Badulin and Shrira, 1999), even in the situations where the system is known for sure to evolve to an attractor, it is often difficult to estimate the characteristic time of this evolution. On the other hand, from the experimental viewpoint, there are two major difficulties of the SBK theory. First, in nature the most often observed crescent-shaped patterns are sporadic and seem to be far from equilibrium. In particular, all patterns observed in wave tank experiments (Su, 1982; Collard and Caulliez, 1999), as well as most of those seen in open-air basins, usually have relatively short (although much exceeding the wave period) time of existence. Second, the patterns have been also observed in the tank experiments without wind (e.g. Su et al, 1982) where no equilibria are possible. All this prompts us to reexamine the basic assumptions of SBK and to look for a new robust mechanism able to create the essentially non-stationary, relatively short-lived patterns of the same geometry.

The present study, being also based on the idea that 
the primary mechanism for the horse-shoes is due to the class II instability of a basic wave, considers general situation when this instability gives rise to many pairs of oblique satellites, with Benjamin-Feir (class I) satellites being also taken into account. Since these questions constitute a problem evidently not tractable by analytical means, an extensive numerical study of possible evolution scenaria was undertaken. For this purpose, a novel numerical method recently developed by the authors (Annenkov and Shrira, 1999) has been used. This method, based on the perturbed integrodifferential Zakharov equation, allows one to trace the long-term evolution of a sufficiently large number of interacting modes, both in a conservative system and in the presence of weak non-conservative effects. Weak dissipation of the satellites, as well as weak generation of the basic wave, was taken into account via the insertion into the numerical model of small (of the order of the five-wave interaction term) linear damping and forcing terms.

The numerical analysis allowed us to establish the basic facts concerned with the long-time evolution of fivewave instability. First, even the system with the initially large number of degrees of freedom appears to follow in most cases, at least locally, the evolution scenario of a low-dimensional system. This means that in a generic situation only very few of linearly unstable modes (often different modes at different moments of time) would grow considerably. Moreover, under the effect of weak dissipation this small number of grown modes is normally reduced to just one pair. This suggests the existence of a nonlinear selection mechanism which is enhanced by dissipation. This mechanism reduces considerably the number of grown modes, at the same time noticeably enlarging their maximal amplitude. Evolution of the system is shown to result in the formation of crescent-shaped transient patterns on the free surface with steep front slopes and flattened rear ones, closely resembling the observed horse-shoe patterns. The sporadic nature of the observed patterns is also reproduced by the simulations. The patterns' dynamics is found to be determined mainly by quintet interactions, while the presence of Benjamin-Feir modulation does not alter it qualitatively.

The selection mechanism has been identified as follows: each growing pair of oblique satellites 'pushes' all other pairs out of the narrow instability domain by creating a nonlinear frequency shift. Normally, one pair prevails, so that most of the time the evolution closely corresponds to that of the three-wave system. At the same time, the presence of other pairs of satellites is shown to lead to phase asymmetry: a growing satellite pair has the required ('correct') phase value, while the phases of decaying ones are unstable, so that the frequency shifts caused by the weak interaction with other modes set them into rapid rotation. As a result, pronounced horse-shoe-like patterns develop at each cycle of the intermittent regime. The described mechanism does not require the presence of non-conservative effects as a prerequisite. Nevertheless, weak dissipation is shown to significantly facilitate observation of the patterns, due to prolonged satellites' growth and elimination of noise.

The paper is organized as follows. Section 2 gives the formulation of the problem in terms of the perturbed Hamiltonian theory of surface waves and describes the numerical procedure employed to solve the Zakharov equation. Section 3 presents the results of simulation of long-time evolution of a system comprising one basic wave and a large number of initially small satellites. We first concentrate on the detailed study of class II instability, while the role of Benjamin-Feir satellites is briefly discussed in Section 5. The field evolution can follow a number of different scenaria; we demonstrate the formation of horse-shoe patterns, both in the conservative and weakly dissipative case, and suggest a qualitative explanation of their properties. An analytic consideration of some simple cases is given. In Section 4 , first, a reappraisal of the familiar case of a basic wave decay into a single pair of oblique satellites is suggested. Then, an asymptotic approach is used to analyse much richer systems comprising two unstable oblique satellites. Section 6 discusses the results and approximations involved. In the Appendix, the model problem of five-wave interactions within the simple three-wave system is considered analytically, and the asymptotic solution for the case of initially small satellites is presented.

\section{Basic equations and the numerical model}

We consider three-dimensional potential gravity waves on the free surface of an incompressible fluid of infinite depth. Wave slopes are supposed to be of the order of a small parameter $\varepsilon$. Dynamics then is governed, up to the order $\varepsilon^{4}$, by the integrodifferential equation

$$
\begin{aligned}
\mathrm{i} \frac{\partial b_{0}}{\partial t}= & \left(\omega_{0}+\mathrm{i} \gamma_{0}\right) b_{0}+\int V_{0123} b_{1}^{*} b_{2} b_{3} \delta_{0+1-2-3} d \mathbf{k}_{123} \\
& +\int W_{01234} b_{1}^{*} b_{2} b_{3} b_{4} \delta_{0+1-2-3-4} \mathrm{~d}_{1234} \\
& +\frac{3}{2} \int W_{43210} b_{1}^{*} b_{2}^{*} b_{3} b_{4} \delta_{0+1+2-3-4} \mathrm{~d} \mathbf{k}_{1234},
\end{aligned}
$$

derived by Zakharov (1968) (see also Crawford et al, 1980) and extended to the order $\varepsilon^{4}$ by Krasitskii (1994); for our purposes, Eq. (1) is modified by the presence of small (of the order $\varepsilon^{4}$ ) non-conservative effects. Here, $b(\mathbf{k})$ is a canonical complex variable, $\omega(\mathbf{k})=(g k)^{1 / 2}$ is the linear dispersion relation, $k=|\mathbf{k}|, \gamma(\mathbf{k})$ stands for $O\left(\varepsilon^{3}\right)$ damping/growth rate, integration in $\mathrm{Eq}$. (1) is performed over the entire $\mathbf{k}$-plane. The compact notation used designates the arguments by indices, e.g., $V_{0123}=V\left(\mathbf{k}, \mathbf{k}_{\mathbf{1}}, \mathbf{k}_{2}, \mathbf{k}_{3}\right), \delta_{0+1-2-3}=\delta\left(\mathbf{k}+\mathbf{k}_{1}-\mathbf{k}_{2}-\mathbf{k}_{3}\right)$, $\mathrm{dk}_{123}=\mathrm{d} \mathbf{k}_{1} \mathrm{~d} \mathbf{k}_{2} \mathbf{d} \mathbf{k}_{3}$, asterisk means complex conjugation, $t$ is time. All the details of the lengthy procedure 
of derivation of Eq. (1), as well as the expressions for the kernels $V, W$ can be found in Krasitskii (1994).

In the conservative case the exact equations of motion can be presented in the equivalent canonical form

$\mathrm{i} \frac{\partial b(\mathbf{k})}{\partial t}=\frac{\delta H}{\delta b^{*}(\mathbf{k})}$,

where the Hamiltonian $H$ is expressed in terms of $b(\mathbf{k})$ by means of integral-power series

$H=\int \omega(\mathbf{k}) b(\mathbf{k}) b^{*}(\mathbf{k}) \mathrm{d} \mathbf{k}+\sum_{n=4}^{\infty} H_{n}$.

The canonical variable $b(\mathbf{k})$ is linked to the Fouriertransformed primitive physical variables $\varphi(\mathbf{k}, t), \eta(\mathbf{k}, t)$ (free-surface potential and elevation of the surface, respectively) also through an integral-power series (Krasitskii, 1994). To a leading order, this relation has the form

$b(\mathbf{k})=\frac{1}{\sqrt{2}}\left\{\sqrt{\frac{\omega(\mathbf{k})}{k}} \eta(\mathbf{k})+\mathrm{i} \sqrt{\frac{k}{\omega(\mathbf{k})}} \varphi(\mathbf{k})\right\}$,

expressions for higher orders generally are very cumbersome. One example of transformation for a particular case is presented in Appendix A of SBK.

The Zakharov equation used in the present work corresponds to the truncation of the series (3) at the term $H_{5}$. The system is then perturbed by adding the terms $\gamma(\mathbf{k})$, small enough to preserve the Hamiltonian structure to the desired order, in a standard way to take into account small non-conservative effects.

Equation (1) has a number of important advantages from the point of view of numerics. First, it is the so called reduced equation, that is, it projects dynamics into the space of nonlinear normal modes of the system, leaving incomparably fewer interactions to consider than in conventional physical space models. In other words, much of the complexity of the original hydrodynamic equations goes to coefficients that indeed have very cumbersome algebraic form. More advantages of Eq. (1) as the basis for a numerical study can be pointed out; one of the most important ones is that the initial discretization is not confined to integer number grids (it does not include Fourier transformation on a timestep), allowing to avoid possible artifacts due to a numerically created resonator (Kartashova, 1991).

Though the idea to use this equation, upon proper discretization, to study the evolution of a number of discrete modes seems natural (Craik, 1985), there were few attempts at implementation of such a numerical scheme. Those known to us (Crawford et al, 1980, repeated in Yuen and Lake, 1987; Krasitskii and Kalmykov, 1993) were aimed at providing only examples of short-term evolution of a few most unstable (usually two-dimensional) modes, often neglecting five-wave interactions and/or not paying respect to the Hamiltonian structure of the equation.
In the present work, the recently proposed novel numerical approach (Annenkov and Shrira, 1999) is used. The algorithm is based on the following strategy. Given the initial state of the fluid (functions $\varphi(\mathbf{x}, 0), \eta(\mathbf{x}, 0)$; where $\mathbf{x}=(x, y))$, Fourier transformation and integral power series expansion are used to obtain the initial value of complex amplitude $b(\mathbf{k}, 0)$. Then the function $b$ is discretized in $\mathbf{k}$-space, being replaced by a set of complex variables $b_{m} \equiv b\left(\mathbf{k}_{m}\right), m=1,2, \ldots N$. Next, all the coefficients $V$ and $W$ are calculated and stored. For the subsequent integration in time, the discretized version of Eq. (1)

$$
\begin{aligned}
\mathrm{i} \frac{\partial B_{m}}{\partial t}= & \mathrm{i} \gamma_{m} B_{m} \\
+ & \sum_{n=1}^{N} \sum_{p=1}^{N} \sum_{q=1}^{N} V_{m n p q} B_{n}^{*} B_{p} B_{q} \times \\
& \times e^{\mathrm{i}\left(\omega_{m}+\omega_{n}-\omega_{p}-\omega_{q}\right) t} \Delta_{m+n-p-q} \\
+ & \sum_{n=1}^{N} \sum_{p=1}^{N} \sum_{q=1}^{N} \sum_{r=1}^{N} W_{m n p q r} B_{n}^{*} B_{p} B_{q} B_{r} \times \\
+ & \quad \frac{3}{2} \sum_{n=1}^{N} \sum_{p=1}^{\mathrm{i}\left(\omega_{m}+\omega_{n}-\omega_{p}-\omega_{q}-\omega_{r}\right) t} \sum_{m+n-p-q-r}^{N} \sum_{r=1}^{N} W_{r q p n m} B_{n}^{*} B_{p}^{*} B_{q} B_{r} \times \\
& \times e^{\mathrm{i}\left(\omega_{m}+\omega_{n}+\omega_{p}-\omega_{q}-\omega_{r}\right) t} \Delta_{m+n+p-q-r}
\end{aligned}
$$

is used, where $B_{m}=b_{m} \exp \left(-\mathrm{i} \omega_{m} t\right), \Delta$ is the Kronecker symbol $\left(\Delta_{m}\right.$ equals to unity when $m=0$ and zero otherwise). Finally, the obtained fluid state $B\left(\mathbf{k}_{m}, t\right)$, $m=1,2, \ldots N$ is transformed back to physical variables.

The outlined scheme is proposed and thoroughly discussed in Annenkov and Shrira (1999), where some examples of its implementation are considered and various impediments are addressed. In particular, the coefficients $V_{m n p q}$ and $W_{m n p q r}$ in Eq. (5) are actually fourand five-dimensional sparse matrices (with a number of symmetry properties), making the construction of an efficient vectorized algorithm to be quite a difficult task. In order to overcome this problem, a special preprocessing routine is included, so that the computed coefficients are arranged into a number of long arrays, and thus all the subsequent operations in Eq. (5) are vectorized. Another difficulty is that the initial discretization implicitly assumes that the wave field at all moments of time is a set of fixed $\delta$-pulses in the wavevector space,

$b(\mathbf{k}, t)=\sum_{m=0}^{N} b_{m}(t) \delta\left(\mathbf{k}-\mathbf{k}_{m}\right)$,

where $\mathbf{k}_{m}$ are constants. However, in reality in course of the field evolution other modes may start to grow from ambient noise. To take this into account, a large number of 'dormant' modes is incorporated into the system: while they do not affect the 'active' modes, they are checked for growth at each timestep using the linearized 
version of Eq. (5). If a dormant mode attains a certain threshold amplitude, it is included into the nonlinear scheme.

When the non-conservative effects are negligible for the timescales under consideration, Eq. (5) is a Hamiltonian system and time integration is performed making use of a symplectic algorithm, otherwise the system is integrated by the more conventional Runge-Kutta scheme.

All the details of the proposed algorithm are given in Annenkov and Shrira (1999). Although it can be used for numerical simulation of wave fields in a variety of surface wave problems, in the present work, we concentrate on its applications to the problem of horse-shoe patterns' formation.

\section{Instability of a single basic wave: evolution scenaria}

In this section we shall try to address a number of fundamental questions concerned with the formation of horseshoe patterns left unanswered by SBK.

As we already mentioned, the model of SBK is confined to the consideration of only three harmonics in the Zakharov equation. Although such an idealisation appears to be supported by the experimental evidence, it does need thorough examination and theoretical justification. In reality, of course, neither the basic wave is monochromatic, nor are the satellites. In this section we will focus upon the issues related to the latter, i.e., we will consider nonlinear evolution of many satellites generated by a single basic wave due to quintet interactions. Among the fundamental open questions addressed are those concerned with the mechanisms of mode selection and the applicability of low-modal approach. (In the present context, we will use the term low-modal as referring to multi-dimensional systems where only a few modes are excited at a time in the process of nonlinear instability.) Similar questions arising in view of the neglect of non-monochromaticity of the basic wave are considered in Sect. 5 .

\subsection{Three-wave model}

First, the numerical method is applied to the simplest three-wave system, in order to recall the basic properties of this important model problem and to discuss in more detail its main limitations.

Consider a system of the form (6) comprising only three waves $(N=3)$ with the amplitudes $a, b, c$ and wavevectors $\mathbf{k}_{a}, \mathbf{k}_{b}, \mathbf{k}_{c}$ satisfying the condition

$3 \mathbf{k}_{a}=\mathbf{k}_{b}+\mathbf{k}_{c}$,

where $a$ denotes the basic wave, $b, c$ are the satellites, assumed initially small, $\mathbf{k}=\left(k_{x}, k_{y}\right)$. Without the loss of generality

$\mathbf{k}_{a}=(1,0), \mathbf{k}_{b}=\left(\frac{3}{2}+p, q\right), \mathbf{k}_{c}=\left(\frac{3}{2}-p,-q\right)$.
It is implied that the waves $a, b, c$ form a nearly resonant quintet, that is

$3 \omega_{a}-\omega_{b}-\omega_{c} \leq O\left(\varepsilon^{3}\right)$.

Thus, this combination of waves represents the simplest possible case of quintet interactions, and the fact that the conservative version of its evolution in time is integrable (Shemer and Stiassnie, 1985) makes it convenient as a test of the algorithm described in the previous section. Eq. (1) for this case takes the form

$$
\begin{aligned}
a_{t}= & -\mathrm{i}\left(\omega_{a}+\mathrm{i} \gamma_{a}\right) a \\
& -\mathrm{i}\left[V_{a a a a}|a|^{2}+2 V_{a b a b}|b|^{2}+2 V_{a c a c}|c|^{2}\right] a \\
& -3 \mathrm{i} W_{b c a a a} b c\left(a^{2}\right)^{*}, \\
b_{t}= & -\mathrm{i}\left(\omega_{b}+\mathrm{i} \gamma_{b}\right) b \\
& -\mathrm{i}\left[2 V_{a b a b}|a|^{2}+V_{b b b b}|b|^{2}+2 V_{b c b c}|c|^{2}\right] b \\
& -\mathrm{i} W_{b c a a a} c^{*} a^{3} \\
c_{t}= & -\mathrm{i}\left(\omega_{c}+\mathrm{i} \gamma_{c}\right) c \\
& -\mathrm{i}\left[2 V_{a c a c}|a|^{2}+2 V_{b c b c}|b|^{2}+V_{c c c c}|c|^{2}\right] c \\
& -\mathrm{i} W_{b c a a a} b^{*} a^{3},
\end{aligned}
$$

System (10) depends on a number of coefficients and three complex amplitudes specified by the initial conditions, and the complete numerical investigation of its dynamics goes beyond the scope of the present work. For our purposes, we will consider only the subset of initial conditions where the satellites are initially very small. As a first meaningful example, the consideration is confined to the case of symmetric satellites, i.e., $\mathbf{k}_{b}=\left(\frac{3}{2}, q_{0}\right), \mathbf{k}_{c}=\left(\frac{3}{2},-q_{0}\right)$, and non-conservative effects are omitted. Then, the conservation of the transverse component of momentum gives

$$
|b|^{2}-|c|^{2}=\text { const. }
$$

\section{Performing the transformation}

$$
a=A \exp (-\mathrm{i} \alpha), b=B \exp (-\mathrm{i} \beta), c=C \exp (-\mathrm{i} \gamma)
$$

it is easy to see that the satellites with initially equal amplitudes remain equal all the time, i.e., $B(t) \equiv C(t)$ provided that $B(0)=C(0)$. In this case, system (10) reduces to the form

$$
\begin{aligned}
A_{t}= & 3 W_{b c a a a} A^{2} B^{2} \sin \Phi \\
B_{t}= & -W_{b c a a a} A^{3} B \sin \Phi \\
\Phi_{t}= & \delta+P A^{2}+M B^{2} \\
& +W_{b c a a a} A\left(9 B^{2}-2 A^{2}\right) \cos \Phi
\end{aligned}
$$

where all the variables are now real, and the two amplitudes are linked by conservation of another component of momentum,

$$
A^{2}+3 B^{2}=I \equiv \text { const }
$$




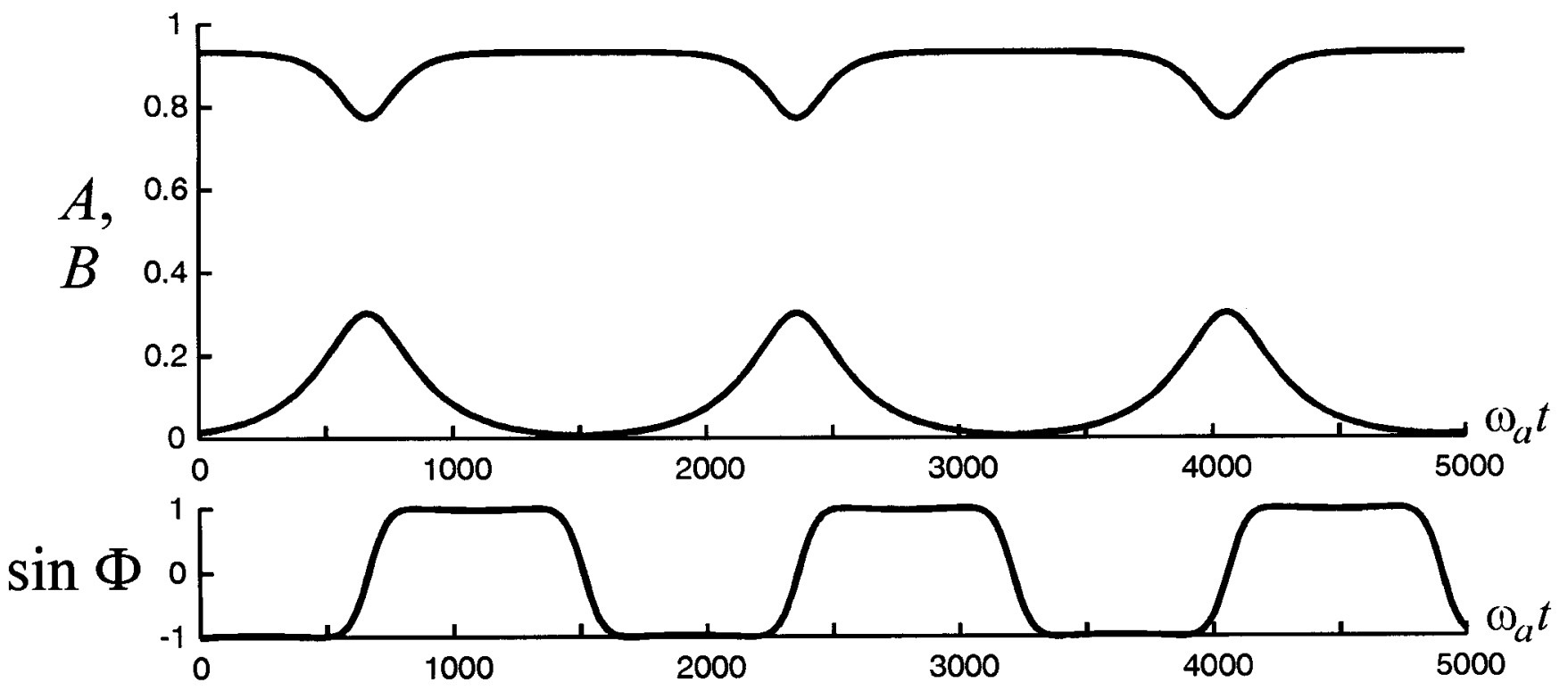

Fig. 1. Evolution of the system of three waves with amplitudes $a, b, c$ and wavevectors $\mathbf{k}_{a}=(1,0), \mathbf{k}_{b}=(1.5,1.52), \mathbf{k}_{c}=(1.5,-1.52)$, obtained by the numerical solution of system (11). Dependence on time of the absolute values of the amplitudes $A(t), B(t)$ and of the phase $\Phi(t)$ (see text for notation) is shown. Initial values are: $A=0.933$ (corresponding to the initial steepness of the basic wave 0.21 ), $B=0.014, \Phi=-\pi / 2$.

while the parameters are

$$
\begin{aligned}
\delta & =3 \omega_{a}-\omega_{b}-\omega_{c} \\
P & =3 V_{a a a a}-2 V_{a b a b}-2 V_{a c a c} \\
M & =6 V_{a b a b}+6 V_{a c a c}-4 V_{b c b c}-V_{b b b b}-V_{c c c c}
\end{aligned}
$$

The variable $\Phi$ is the phase of the pair of satellites relative to the fundamental and is defined as

$\Phi=3 \alpha-\beta-\gamma$,

from now on, for brevity, we will refer to this value (and sometimes also to its sine) simply as 'the phase'.

The specific form of a surface elevation pattern is mainly prescribed by the value of $\Phi$. If $-\pi+2 \pi n<\Phi<$ $0+2 \pi n$, the patterns have their convex sides oriented downwind, the best resemblance with the observed patterns being at $\Phi=-\pi / 2+2 \pi n$. For simplicity we will refer to such phases as negative and will omit the period $2 \pi n$ hereafter. Evidently, phases in the range $0<\Phi<\pi$ correspond to the opposite orientation. However, as was noted by SBK, Eqs. (11) form a reversible Hamiltonian system, so that $\Phi$ passes in a symmetric manner through all its values. Thus, the system shows a variety of threedimensional patterns with no preference for orientation. This means that a model of horse-shoe patterns cannot be built upon the basis of such a conservative threewave system. SBK went on to incorporate small nonconservative effects into system (11) and showed that attractive equilibria with the required properties do appear.

Still, a closer inspection of this system is useful for further progress in understanding. If the initial amplitudes of the satellites are infinitesimal, then the trajectories of system (11) are close to the separatrix originating and ending at two stationary saddle points

$$
B=0, A=A_{0}, \Phi=\Phi_{0}= \pm \cos ^{-1} \frac{\delta+P A_{0}^{2}}{2 W A_{0}^{3}}
$$

For definiteness, and also because this ensures the maximal instability (see Stiassnie and Shemer, 1987), we choose $\delta=-P A_{0}^{2}$, and then $\Phi_{0}= \pm \pi / 2$. An example of a triad evolution for such a case is presented in Fig. 1, for the initial value of phase $\Phi$ equal to $-\pi / 2$. The $d y$ namics is periodic, with the recurrence period tending to infinity when the initial satellite amplitude tends to zero (Stiassnie and Shemer, 1987). The phase, rotating counterclockwise, most of time stays close to $\Phi=-\pi / 2$ (growth of satellites) or $\Phi=\pi / 2$ (decay) with quite fast transition between these values, zero phase corresponding to the point of the deepest modulation. When the satellites are small compared to the fundamental, all the trajectories in $(B, \Phi)$-plane are in the vicinity of the saddle points. Since the trajectories in the vicinity of the saddle $(B=0, \Phi=-\pi / 2)$ are divergent in $B$, there is convergence of the trajectories in $\Phi$, while in the neighbourhood of the second saddle $(B=0, \Phi=\pi / 2)$ there is convergence in $B$ but divergence in $\Phi$. This implies that if we perturb the system the trajectories corresponding to growing satellites will tend to preserve their phase, while those corresponding to decaying satellites will experience large variations of the phase, and thus, since the system dynamics is very sensitive to the phase, become unstable. 


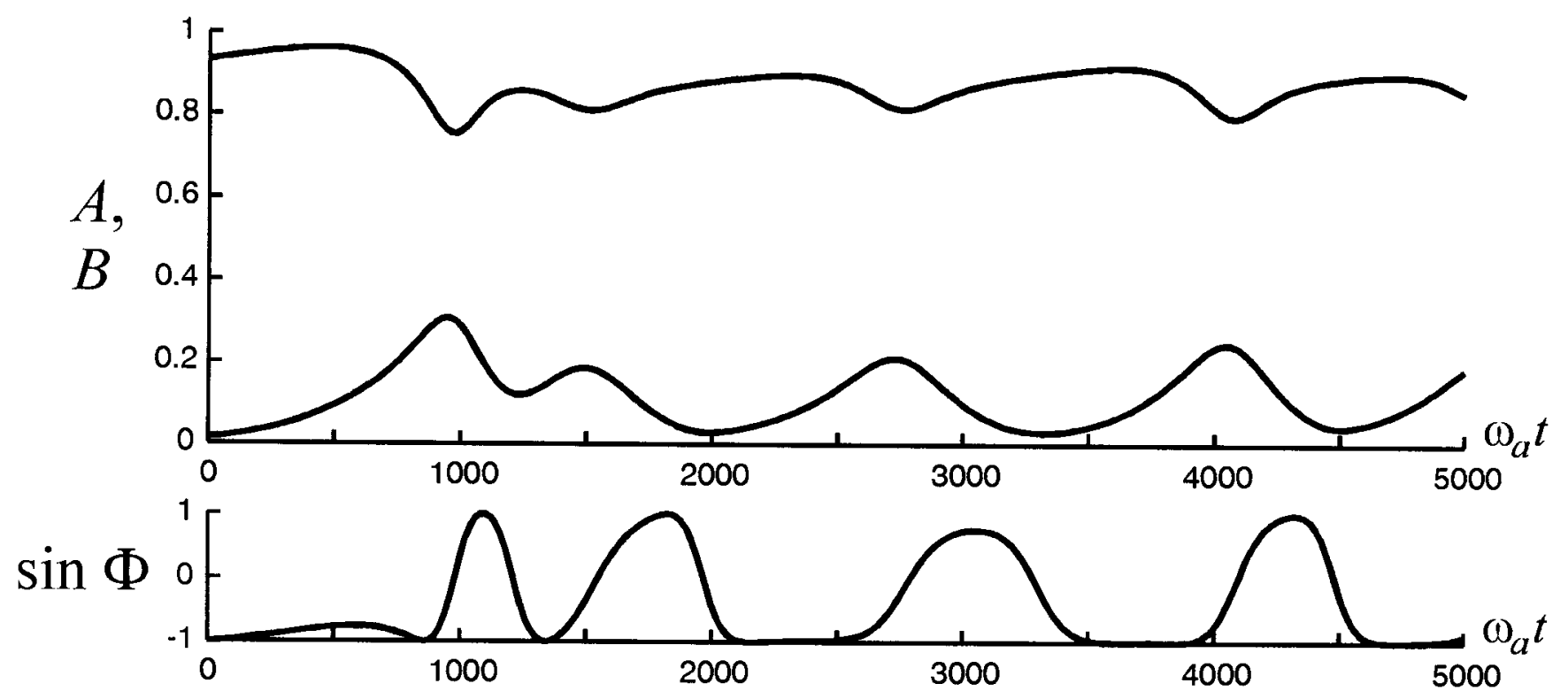

Fig. 2. Evolution of the system of three waves with amplitudes $a, b, c$ and wavevectors $\mathbf{k}_{a}=(1,0), \mathbf{k}_{b}=(1.5,1.52), \mathbf{k}_{c}=(1.5,-1.52)$, obtained by the numerical solution of non-conservative system (13), with weak forcing of the basic wave $\Gamma_{a}=5 \cdot 10^{-5} \omega_{a}$ and weak dissipation of the satellites $\Gamma_{b}=\Gamma_{c}=-5 \cdot 10^{-4} \omega_{b}$. All the other parameters and initial values are as in Fig. 1.

These simple facts give insight into more complicated situations. Although within the framework of Eqs. (11) the phase stays equal time in the vicinity of these points, even a small perturbation can change the phase and thus the whole system dynamics near the point $\Phi=+\pi / 2$. In other words, the long periods in the neighbourhood of $\Phi=+\pi / 2$ and potential instability of these segments of trajectories indicate a possible structural instability of the three-wave system.

With the inclusion of small, in the above-mentioned sense, non-conservative effects the evolution of the threewave system is controlled by (see SBK)

$$
\begin{aligned}
A_{t}= & 3 W_{b c a a a} A^{2} B^{2} \sin \Phi+\Gamma_{a} A, \\
B_{t}= & -W_{b c a a a} A^{3} B \sin \Phi+\Gamma_{b} B, \\
\Phi_{t}= & \delta+P A^{2}+M B^{2} \\
& +W_{b c a a a} A\left(9 B^{2}-2 A^{2}\right) \cos \Phi .
\end{aligned}
$$

where $\Gamma_{a}=\omega_{a} \gamma_{a}, \Gamma_{b}=\omega_{b} \gamma_{b}$. For wind waves, it is natural to assume that $\Gamma_{a}>0, \Gamma_{b}<0$ (weak generation of the fundamental and weak dissipation of the satellites). Note that if $\Gamma_{c} \cong \Gamma_{b}$, then

$$
\left(|b|^{2}-|c|^{2}\right)_{t}=\Gamma_{b}\left(|b|^{2}-|c|^{2}\right)
$$

that is, $\left(|b|^{2}-|c|^{2}\right)$ decays exponentially with the rate $\Gamma_{b}$, so that the transverse symmetry adopted in the derivation of (13) is structurally stable. If we again consider the dynamics of satellites on $(B, \Phi)$-plane assuming them small compared to the fundamental and neglecting variations of the latter, the truncated system (13) with the first equation for $A$ omitted preserves the similar two saddles. The typical evolution scenario for initially small amplitudes of the satellites is portrayed in Fig. 2. The evolution becomes slightly asymmetrical since the growth of satellites is slower and their subsequent decay is faster, as it follows straightforwardly from the linearized version of (13). At the point of maximum, the phase is negative. This regime corresponds to a limit cycle in the phase space of (13). Other more complicated regimes, e.g., limit cycle with period doubling, quadrupling, etc, are also possible but far less typical (see Badulin and Shrira, 1999). The plot (Fig. 2), in particular, illustrates the fact that there are still relatively long periods when the phase stays close to the potentially unstable value $+\pi / 2$, suggesting that the non-conservative three-wave system remains potentially structurally unstable and thus might be too oversimplified to serve as a realistic model of the formation of horse-shoe patterns.

\subsection{Multiple class II satellites}

In reality, a fundamental wave of finite amplitude possesses finite size domains of instability with respect to four- and five-wave processes in the wavevector space (see McLean, 1982; Craik, 1985, Fig. 6.6), so that the simultaneous growth of many pairs of satellites (strictly speaking, continuum of satellites) should occur. Nonlinear dynamics of such a continuum of linearly growing satellites is simulated within the framework of the discretized system (5) by a large number of unstable satellites. Since our primary interest is in three-dimensional dynamics, in this section we focus upon the situations where the satellites were taken in the five-wave (class II) instability domain and the stable part of the $\mathbf{k}$-plane 


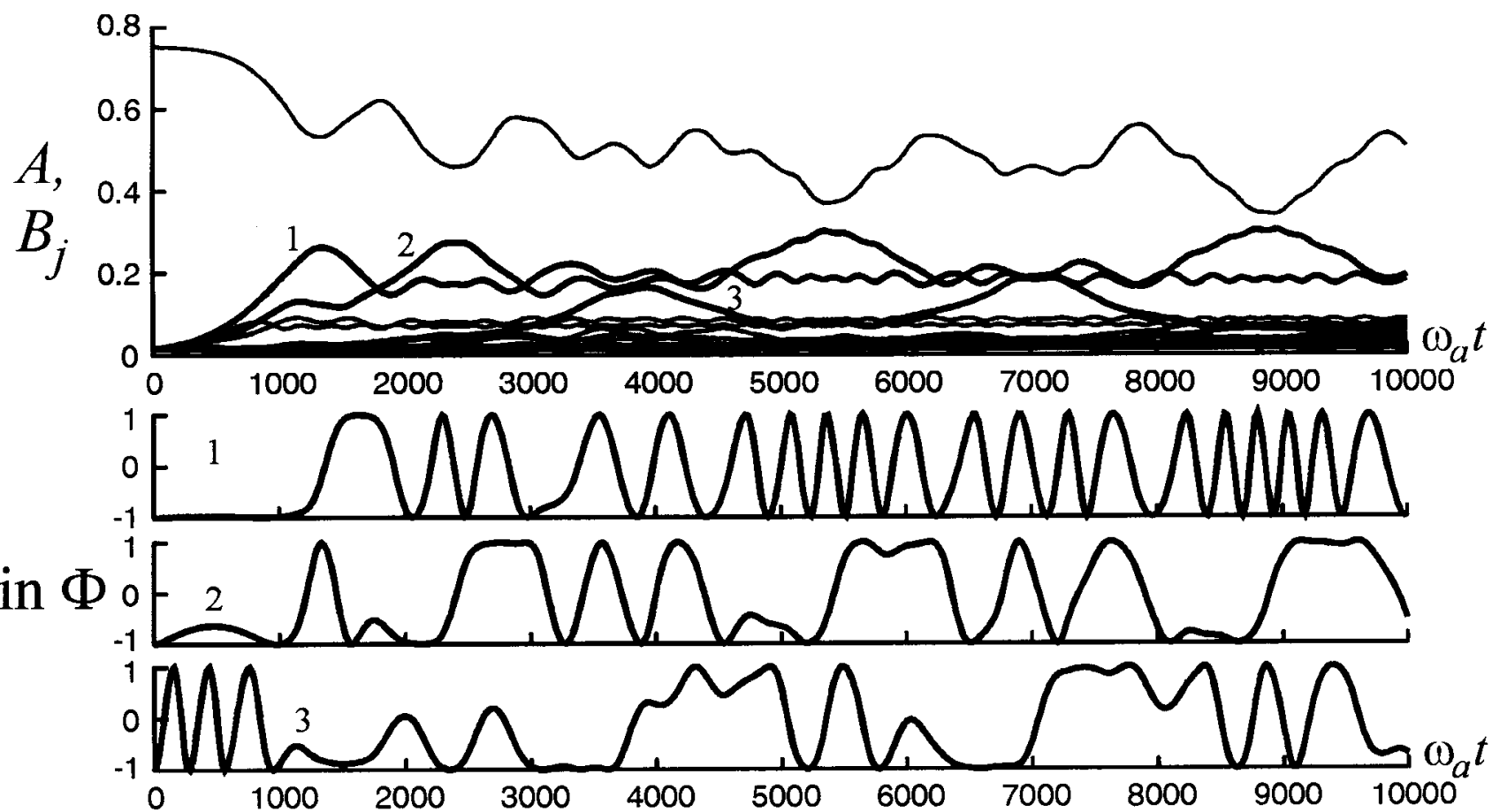

Fig. 3. Evolution of the system of 85 waves obtained by the numerical solution of the conservative Zakharov equation. The system consists of the basic wave, with initial amplitude $A(0)=0.755$ (corresponding to steepness 0.17 ), and 42 pairs of initially small $\left(B_{j}(0)=0.014\right.$, $j=1,2, \ldots, 84)$ satellites, chosen randomly in $\mathbf{k}$-plane according to condition (14). For three most unstable pairs (plotted with bold curves and numbered $1,2,3$ in the amplitude plot), evolution of phase is shown.

only. The effect of finite bandwidth of the basic wave spectrum and, in particular, role of the Benjamin-Feir instability is briefly addressed in Sect. 5 .

For the numerical study, a system comprising a basic wave with wavevector $\mathbf{k}_{0}=(1,0)$ and $N$ pairs of oblique satellites with wavevectors $\mathbf{k}_{j, j+1}=\left(\frac{3}{2} \pm p_{j}, \pm q_{j}\right), j=$ $1,2, \ldots, N$ was selected. In order to represent both symmetric (with respect to $k_{x}$-axis) and non-symmetric oblique harmonics within and in the neighbourhood of the instability domain, condition

$$
\begin{array}{ll}
p_{j}=0, & 1 \leq j \leq \frac{N}{2} \\
1<p_{j}<2, \quad p_{j} \neq 0, & \frac{N}{2}<j \leq N \\
1.48 \leq q_{j} \leq 1.68 & 1 \leq j \leq N
\end{array}
$$

was imposed; in the experiments discussed in this section, $N=42$. Three different values for the initial steepness of the fundamental $(0.13,0.17$ and 0.21$)$ were used. The satellites were put initially small, with the amplitudes of the order $O\left(10^{-2}\right)$ relative to the amplitude of the fundamental; no essential dependence on the exact value of satellites' initial amplitude was revealed. Initial phases of all the satellites were again prescribed at the value most favourable for growth $(-\pi / 2)$. Evolution in time was traced for about $10^{3}$ periods of the basic wave. We again emphasize that at this stage the Benjamin-Feir instability was excluded.
An example of the conservative evolution of the system is presented in Fig. 3. The plot demonstrates two distinct features of the class II instability with respect to multiple satellites. First, most of the modes located in the linear instability domain do not attain considerable amplitudes; instead, their growth is quickly arrested, resulting in stagnation at a quite low level. Only a few modes at each moment can grow inattenuated, though this behaviour is displayed by different modes at different moments. Second, the evolution of the phases of these growing modes differs noticeably from that of the isolated three-wave system. In particular, it becomes essentially asymmetric. While a mode is growing, its phase keeps close to $-\pi / 2$, as in the three-mode case; the maximum of the satellite amplitude again corresponds to zero phase, but soon after reaching the maximum of the amplitude, the phase typically starts to change much more rapidly, while the amplitude quickly decreases and tends to stagnate at a low level. It can be noticed, however, that the described behaviour is not always well pronounced for all growing pairs during the course of the conservative evolution, thus allowing one to consider it only as a tendency.

Meanwhile, in the more realistic weakly non-conservative case (Fig. 4), these features are much better pronounced. The phases of a few growing satellites are close to $-\pi / 2$ during their growth and near the maximum, while during the decay the phases rapidly change. For 


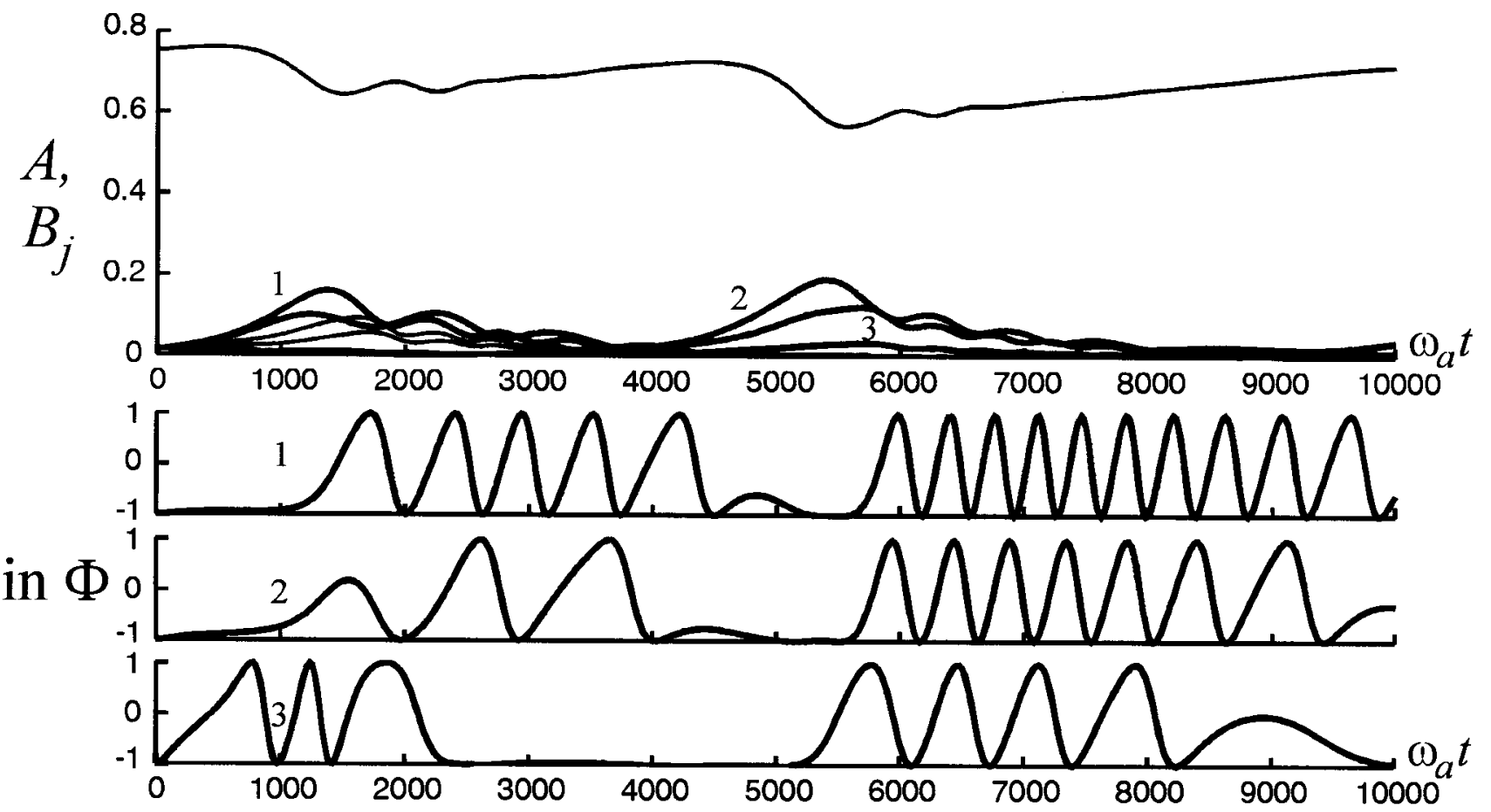

Fig. 4. Evolution of the system of 85 waves obtained by the numerical solution of the Zakharov equation perturbed by small nonconservative effects. The system is the same as in Fig. 3, with weak forcing for the basic wave and weak dissipation for the satellites $\left(\Gamma_{0}=5 \cdot 10^{-5} \omega_{0}, \Gamma_{j}=-5 \cdot 10^{-4} \omega_{j}, j=1,2, \ldots, N\right)$.

somewhat larger initial basic wave steepness (Fig. 5), after the initial growth of all linearly unstable modes, only one pair of satellites attains considerable amplitude, while its phase remains in the neighbourhood of $-\pi / 2$ nearly all the time, except for the brief excursion soon after the maximum of amplitude. Overall, the evolution of the satellites can be summarized as follows:

(i) at each particular moment maximum one pair is large, though at different moments different modes may prevail;

(ii) when a satellite pair starts to grow, its phase is set close to $-\pi / 2$ and remains in its vicinity almost until the maximum of the satellite amplitude is reached; at the maximum of the amplitude the phase is still negative;

(iii) after the mode passes the point of its maximum amplitude, the phase begins to change (rotate) rapidly;

(iv) if a satellite decays or does not grow, its phase rotates passing quickly through all possible values.

These features of the behaviour were observed in the large number of runs of the numerical model.

\subsection{Two unstable pairs}

The non-trivial dynamics demonstrated above for the case of the basic wave instability with respect to multiple class II satellites requires a more profound study. For this purpose, consider first, as the simplest non-trivial model, a five-wave system with just two pairs of satellites, using the notation $\left(\mathbf{k}_{b}, \mathbf{k}_{c}\right)$ for the first pair and $\left(\mathbf{k}_{d}, \mathbf{k}_{e}\right)$ for the second one, so that

$$
3 \mathbf{k}_{a}=\mathbf{k}_{b}+\mathbf{k}_{c}=\mathbf{k}_{d}+\mathbf{k}_{e}
$$

and the frequency mismatches $3 \omega_{a}-\omega_{b}-\omega_{c}$ and $3 \omega_{a}-$ $\omega_{d}-\omega_{e}$ are both of the order of $\varepsilon^{3}$. Again, it is convenient to assume that

$$
\begin{aligned}
& \mathbf{k}_{a}=(1,0) \\
& \mathbf{k}_{b}=\left(\frac{3}{2}+p_{1}, q_{1}\right), \mathbf{k}_{c}=\left(\frac{3}{2}-p_{1},-q_{1}\right) \\
& \mathbf{k}_{d}=\left(\frac{3}{2}+p_{2}, q_{2}\right), \mathbf{k}_{e}=\left(\frac{3}{2}-p_{2},-q_{2}\right)
\end{aligned}
$$

Introducing, as above, the transformation

$$
\begin{aligned}
a & =A \exp (-\mathrm{i} \alpha), \\
b & =B \exp \left(-\mathrm{i} \beta_{1}\right), c=B \exp \left(-\mathrm{i} \gamma_{1}\right), \\
d & =D \exp \left(-\mathrm{i} \beta_{2}\right), e=D \exp \left(-\mathrm{i} \gamma_{2}\right),
\end{aligned}
$$

we arrive at the system of equations of the form

$$
\begin{aligned}
A_{t}= & 3 W_{b c a a a} A^{2} B^{2} \sin \Phi_{1} \\
& +3 W_{\text {deaaa }} A^{2} D^{2} \sin \Phi_{2}+\Gamma_{a} A, \\
B_{t}= & -W_{b c a a a} A^{3} B \sin \Phi_{1} \\
& +2 V_{b c d e} B D^{2} \sin \Psi+\Gamma_{b} B
\end{aligned}
$$




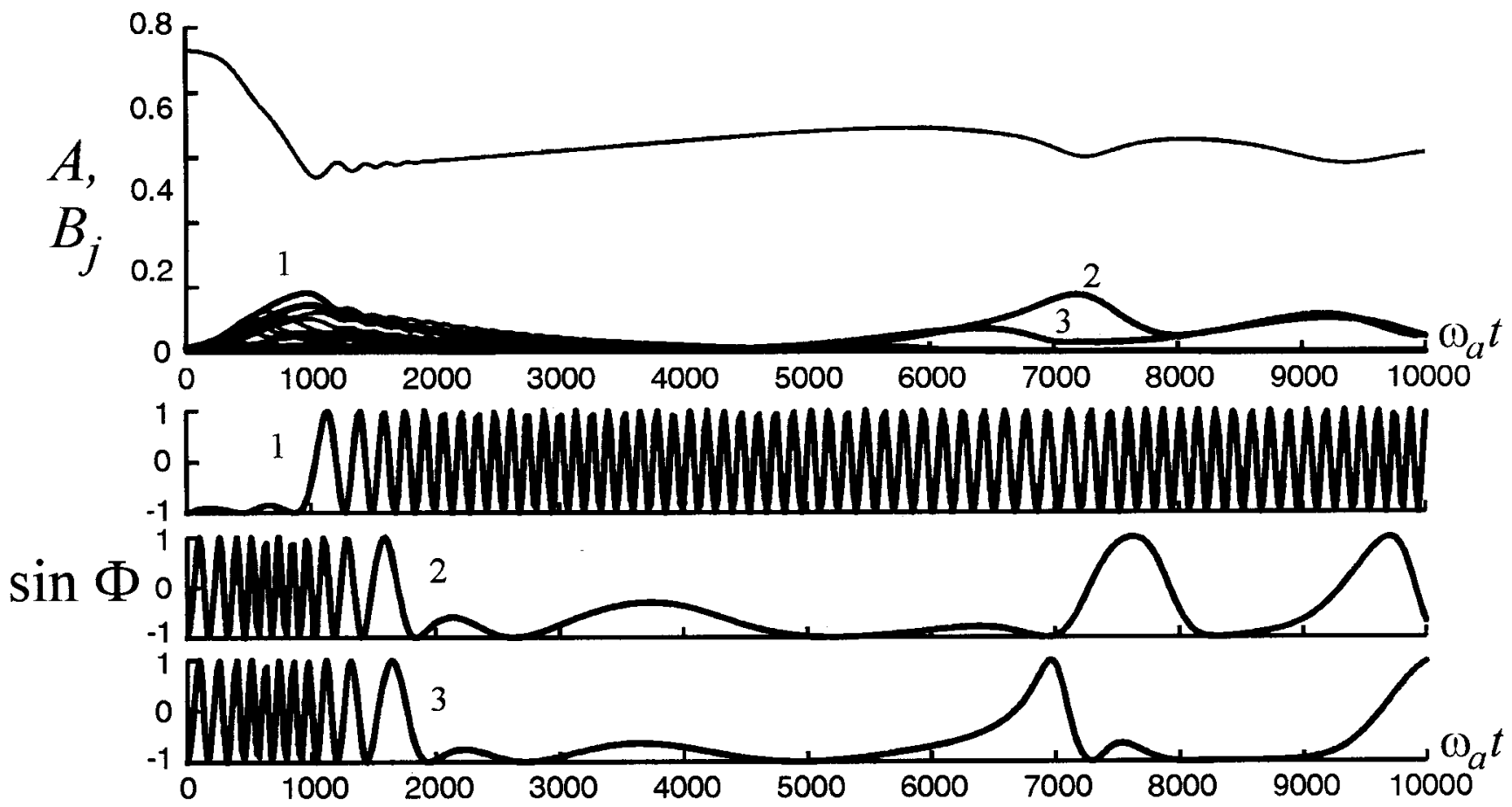

Fig. 5. As in Fig. 4, for larger initial steepness of the basic wave $(A(0)=0.933$, corresponding to steepness 0.21$)$.

$$
\begin{aligned}
D_{t}= & -W_{\text {deaaa }} A^{3} D \sin \Phi_{2} \\
& -2 V_{b c d e} D B^{2} \sin \Psi+\Gamma_{d} D \\
\Phi_{1 t}= & \delta_{1}+P_{1} A^{2}+M_{1} B^{2}+\left(N_{1}-4 V_{b c d e} \cos \Psi\right) D^{2} \\
& +W_{b c a a a} A\left(9 B^{2}-2 A^{2}\right) \cos \Phi_{1} \\
& +9 W_{\text {deaa } a} A D^{2} \cos \Phi_{3} \\
\Phi_{2 t}= & \delta_{2}+P_{2} A^{2}+M_{2} D^{2}+\left(N_{2}-4 V_{b c d e} \cos \Psi\right) B^{2} \\
& +W_{\text {deaaa }} A\left(9 D^{2}-2 A^{2}\right) \cos \Phi_{2} \\
& +9 W_{b c a a a} A B^{2} \cos \Phi_{1} \\
& \delta_{3}+P_{3} A^{2}+\left(N_{3}-4 V_{b c d e} \cos \Psi\right) B^{2} \\
& +\left(N_{4}+4 V_{b c d e} \cos \Psi\right) D^{2} \\
& +2 A^{3}\left(W_{b c a a a} \cos \Phi_{1}-W_{\text {deaa }} \cos \Phi_{2}\right)
\end{aligned}
$$

where

$$
\begin{aligned}
\Phi_{1} & =3 \alpha-\beta_{1}-\gamma_{1}, \Phi_{2}=3 \alpha-\beta_{2}-\gamma_{2} \\
\Psi & =\beta_{1}+\gamma_{1}-\beta_{2}-\gamma_{2} \\
\delta_{1} & =3 \omega_{a}-\omega_{b}-\omega_{c}, \delta_{2}=3 \omega_{a}-\omega_{d}-\omega_{e} \\
\delta_{3} & =\omega_{b}+\omega_{c}-\omega_{d}-\omega_{e}
\end{aligned}
$$

and the coefficients are

$$
\begin{aligned}
P_{1}= & 3 V_{a a a a}-2 V_{a b a b}-2 V_{a c a c} \\
M_{1}= & 6 V_{a b a b}+6 V_{a c a c} \\
& -4 V_{b c b c}-V_{b b b b}-V_{c c c c} \\
N_{1}= & 6 V_{a d a d}+6 V_{a e a e} \\
& -2 V_{b d b d}-2 V_{b e b e}-2 V_{c d c d}-2 V_{c e c e}
\end{aligned}
$$

Expressions for $P_{2}, M_{2}, N_{2}$ can be obtained in the same way, transposing $b \leftrightarrow d$ and $c \leftrightarrow e$. Expressions for coefficients in the equation for $\Psi$ will not be used and are omitted.

As previously, the initial values of $\Phi_{1}$ and $\Phi_{2}$ are assumed equal to $-\pi / 2$, corresponding to the maximal instability rate. Since both pairs lie in the instability domain, it is obvious that the evolution begins with their simultaneous growth, as predicted by the linear theory. As the satellites attain a certain amplitude (still much smaller than that of the basic wave), nonlinear effects become significant, and the further evolution depends on specific combinations of parameters. The main conclusion is that the presence of extra pairs of satellites of initially small (but not infinitesimal) amplitudes can change significantly the dynamics of an isolated triad.

Simulations show that in most cases the simultaneous growth of both pairs up to considerable values of amplitude does not occur. Instead, the modes grow in alternance (Fig. 6): after the initial linear stage of instability one of harmonics stagnates at a quite low amplitude level, while the other one continues to grow, attains considerable amplitude and decays similar to the evolution of the three-wave system. At this stage, the presence of another pair of harmonics does not seem to affect the system behaviour at all, except for a rather remarkable fact that the maximum of amplitude of the growing pair in the presence of the stagnating one reaches higher values than in its absence. Further on, the process is approximately repeated with another pair of satellites, so that most of time the evolution appears to be close to that of the three-wave system. 


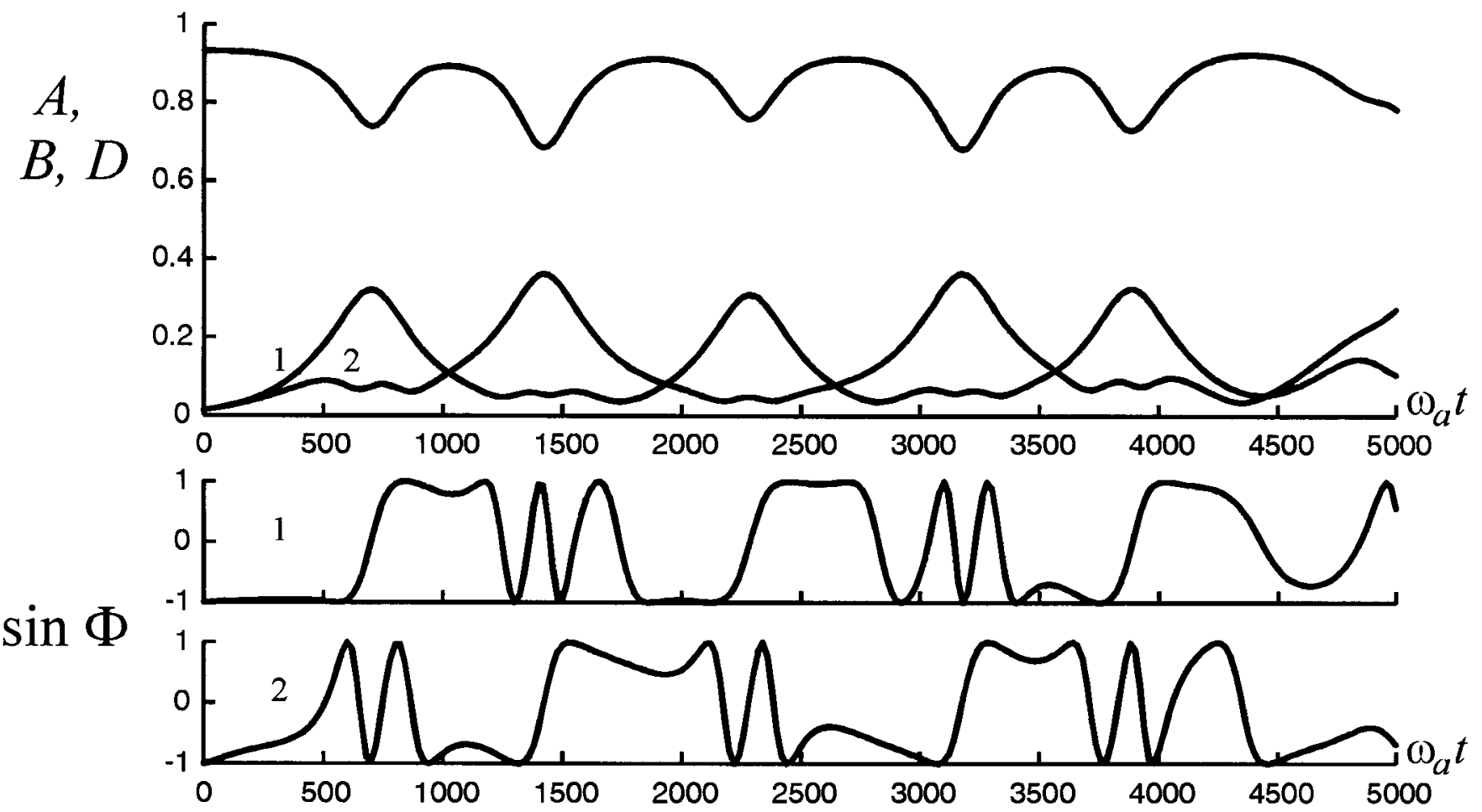

Fig. 6. Example of the regime of evolution of five-wave system (15). Evolution of the system of five waves with amplitudes $a, b, c, d$, $e$ and wavevectors $\mathbf{k}_{a}=(1,0), \mathbf{k}_{b}=(1.5,1.542), \mathbf{k}_{c}=(1.5,-1.542), \mathbf{k}_{d}=(1.5,-1.555), \mathbf{k}_{e}=(1.5,-1.555)$ is shown. Dependence on time of the absolute values of all amplitudes (above) and of the phases $\Phi_{1}(t), \Phi_{2}(t)$ (below) is plotted. Non-conservative effects are not included. Initial values are: $A=0.933$ (corresponding to the initial steepness of the basic wave 0.21 ) $, B=D=0.014, \Phi_{1}=\Phi_{2}=-\pi / 2$.

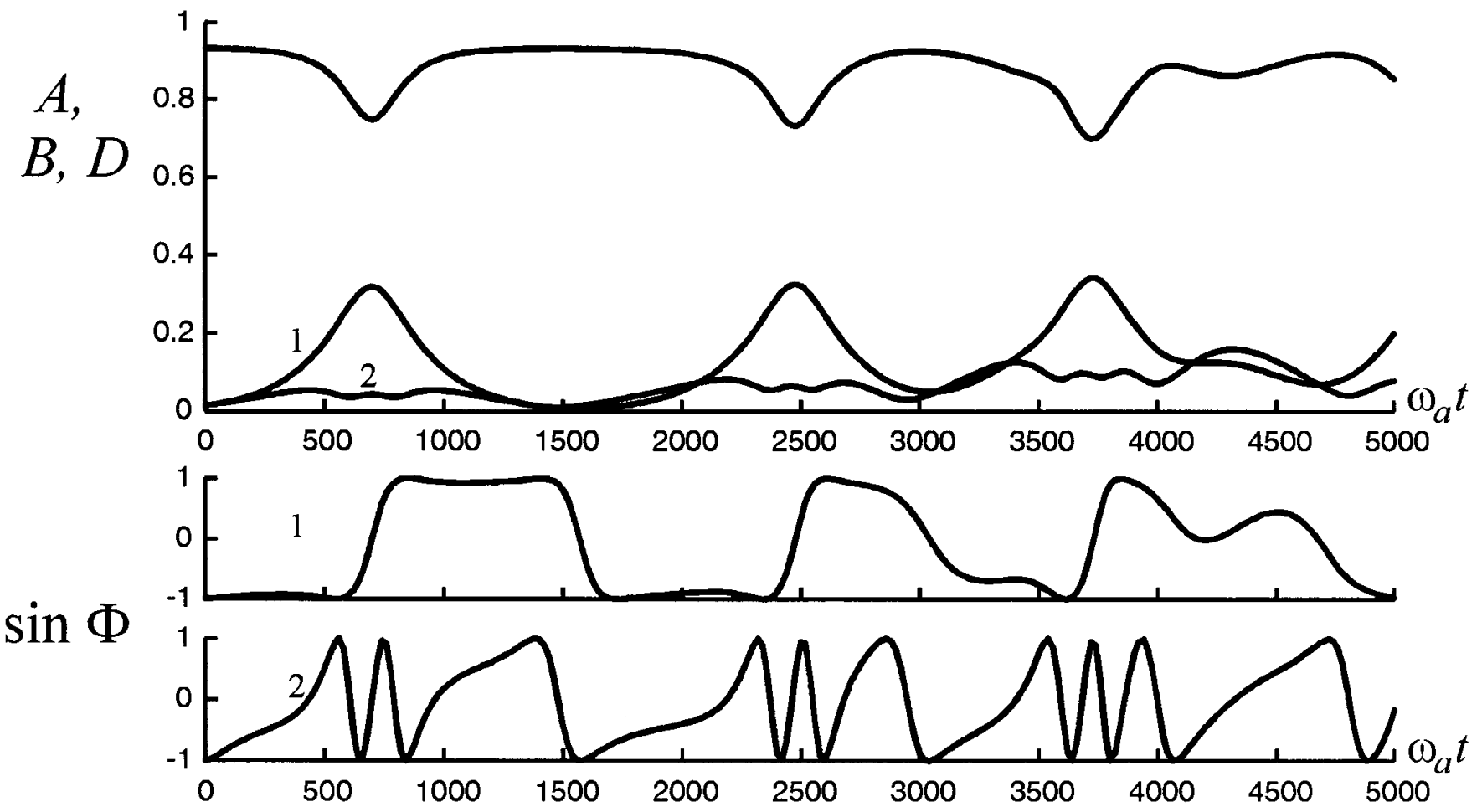

Fig. 7. Another example of the regime of evolution of five-wave system (15). Wavevectors are chosen as $\mathbf{k}_{a}=(1,0), \mathbf{k}_{b}=(1.5,1.52)$, $\mathbf{k}_{c}=(1.5,-1.52), \mathbf{k}_{d}=(1.5,-1.55), \mathbf{k}_{e}=(1.5,-1.55)$, all the other parameters are as in Fig. 6. 


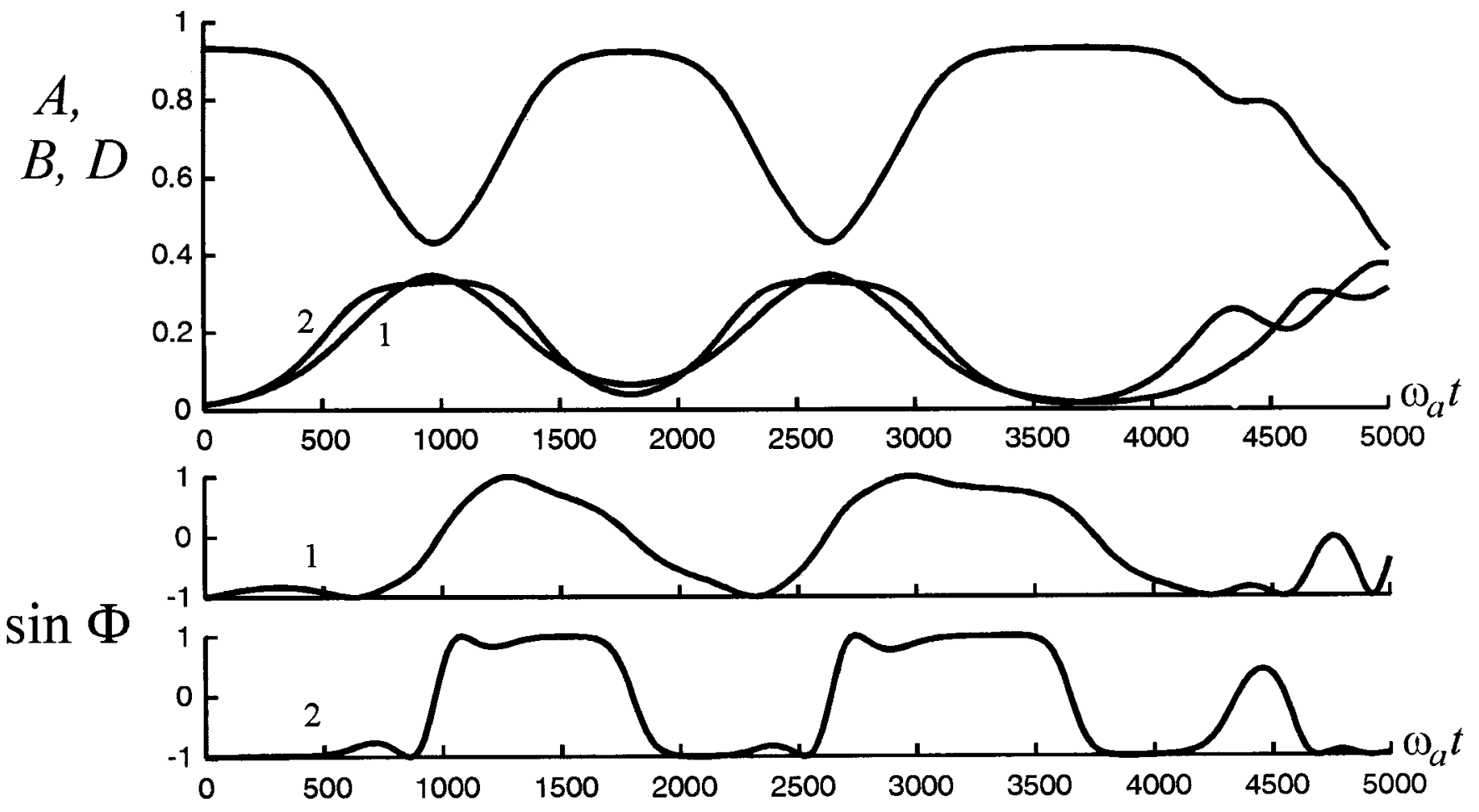

Fig. 8. Third example of the regime of evolution of five-wave system (15). Wavevectors are chosen as $\mathbf{k}_{a}=(1,0)$, $\mathbf{k}_{b}=(1.5,1.52)$, $\mathbf{k}_{c}=(1.5,-1.52), \mathbf{k}_{d}=(1.5,-1.53), \mathbf{k}_{e}=(1.5,-1.53)$, all the other parameters are as in Fig. 6.

This scenario of evolution normally takes place when both pairs are close to the maximum of linear instability (see Sect. 4). Meanwhile, if one of the pairs is characterized by somewhat higher linear growth rate, it can suppress the growth of another pair nearly completely (Fig. 7).

In an exceptional case when all the satellites lie at the margin of the linear instability domain corresponding to the lowest values of the transverse wavenumber $q$, where the growth rates are relatively small, the third possible scenario is observed (Fig. 8). Both pairs grow simultaneously and both attain higher amplitudes than in the absence of each other.

\subsection{Summary and discussion}

We will now summarize the results of simulations referring to the instability of a basic wave with respect to many pairs of oblique initially small satellites. While three-wave systems, with small non-conservative effects, exhibit nearly symmetric behaviour with respect to different values of phase, inclusion of one or more additional pairs of satellites drastically enhances the asymmetry. The phase of a satellite during its growth and near the maximum of amplitude remains close to $-\pi / 2$; the phase of the decaying one in most cases becomes indeterminate, especially under the presence of small dissipation effects.

The important role of dissipation requires some spe- cial comments. First, the dissipation is essential in the initial selection, since only the modes with maximal growth rates and initial phases close to $-\pi / 2$ can survive. Second, the presence of dissipation results in shifting the phase at the point of maximal amplitude of the satellite: the phase remains negative at the maximum and in its neighbourhood. Thus, the phases of modes whose amplitudes exceed a certain threshold are always negative, and therefore the wave fronts are oriented forward. Such sporadically appearing and disappearing surface patterns closely resemble the observed horse-shoe ones. The main contribution to the phase and, thus, to the front curvature asymmetry comes from the apparent instability of the trajectories for negative phases, as discussed earlier.

The most nontrivial fact established numerically is that the evolution of a multi-dimensional system remains effectively low-dimensional. This is quantitatively characterized in the histograms in Figs. 9, 10 and merits some discussion. First we emphasize that the observed low-dimensionality of dynamics is partly due to our very special choice of variables which excludes all 'slave' modes retaining only true active modes. The second factor, expected to contribute to the low-dimensionality, is the phase volume contraction typical of dissipative systems. Indeed, it is well known that a nonlinear system characterized at an initial moment by a certain finite number of excited modes can evolve in accordance with two opposite scenaria. If nonlinearity prevails (in 

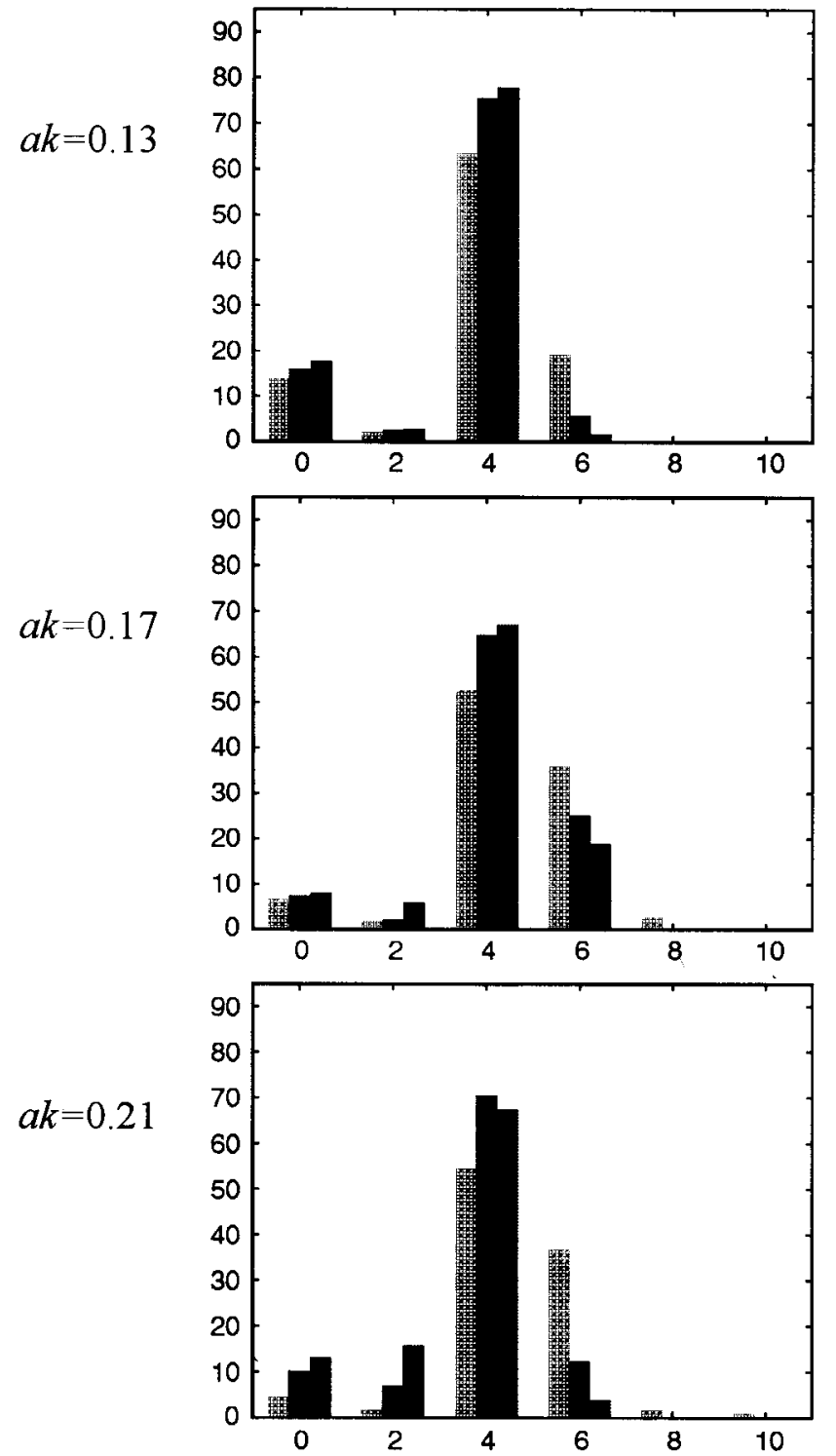

Fig. 9. Percentage of time (vertical axis), during which the specified number of grown satellites (horizontal axis) is present in the conservative system. All the parameters of the system are the same as in Fig. 3, except for the basic wave steepness (three different values are used). Evolution was traced up to 10000 time units (inverse values of the basic wave frequency). For the purpose of these histograms, a satellite was considered grown if its energy exceeded a certain level relative to the total energy of the system: $2 \%$ (light bars), $4 \%$ (medium bars), $6 \%$ (dark bars). a certain sense) over dissipative effects, the number of excited modes of the system tends to grow with time, in the opposite limit the effective number of active modes in the system decreases and the system often falls into a low-modal attractor. At first sight the latter scenario seems to be the case. It is easy to find the contraction rate $\nu$ of the phase volume

$$
\nu=\Gamma_{a}+\sum_{j=1}^{N} \Gamma_{b_{j}}+\sum_{j=1}^{N} \Gamma_{c_{j}}
$$

and it is indeed negative when the integral dissipation $\left|\sum_{j=1}^{N} \Gamma_{b_{j}}+\sum_{j=1}^{N} \Gamma_{c_{j}}\right|$ exceeds the input $\Gamma_{a}$. This is the case for all our simulations when non-conservative effects were taken into account. However, the distinct tendency towards low-dimensionalisation of dynamics is revealed even in the case of purely Hamiltonian dynamics, prompting to conclude that non-conservative effects are of secondary importance in this context. Though this seems to contradict the phase volume preservation, the contraction of the phase volume in physically important segments of the phase space is exactly compensated by its expansion in other segments. For instance, confinement of the phases of the growing and grown modes is accompanied by the rapid phase rotation of all other modes. Strictly speaking, in the purely conservative case the number of modes in the system actually grows, in particular, due to neglected here quartet interactions. However, this growth occurs in the subspace of the phase space of no physical interest in the present context. If one focuses attention on the subspace of interest, say the modes with amplitudes exceeding a certain small threshold, the rest of the phase space will provide a kind of nonlinear dissipation for the chosen subspace.

Turning back to the observed phenomena, we note that in most cases, provided that weak dissipation is taken into account, we have seen that the multiple satellite system most of the time can be considered as a perturbation of a certain three-wave system (different at different moments): at each moment only one pair of satellites can attain considerable amplitude, while all the other ones are small and can be treated as a perturbation. This raises hope to build a plausible model of the instability of a basic wave with respect to a large number of oblique satellites on the basis of matched asymptotic expansions. Construction of such a theory lies beyond the scope of the present work; nevertheless, in the next section we will discuss a model of the selection mechanism, based on this idea.

\section{Selection mechanism}

Numerical results discussed in the previous section suggest that there exists a certain nonlinear mechanism responsible for the selection of modes and the eventual 

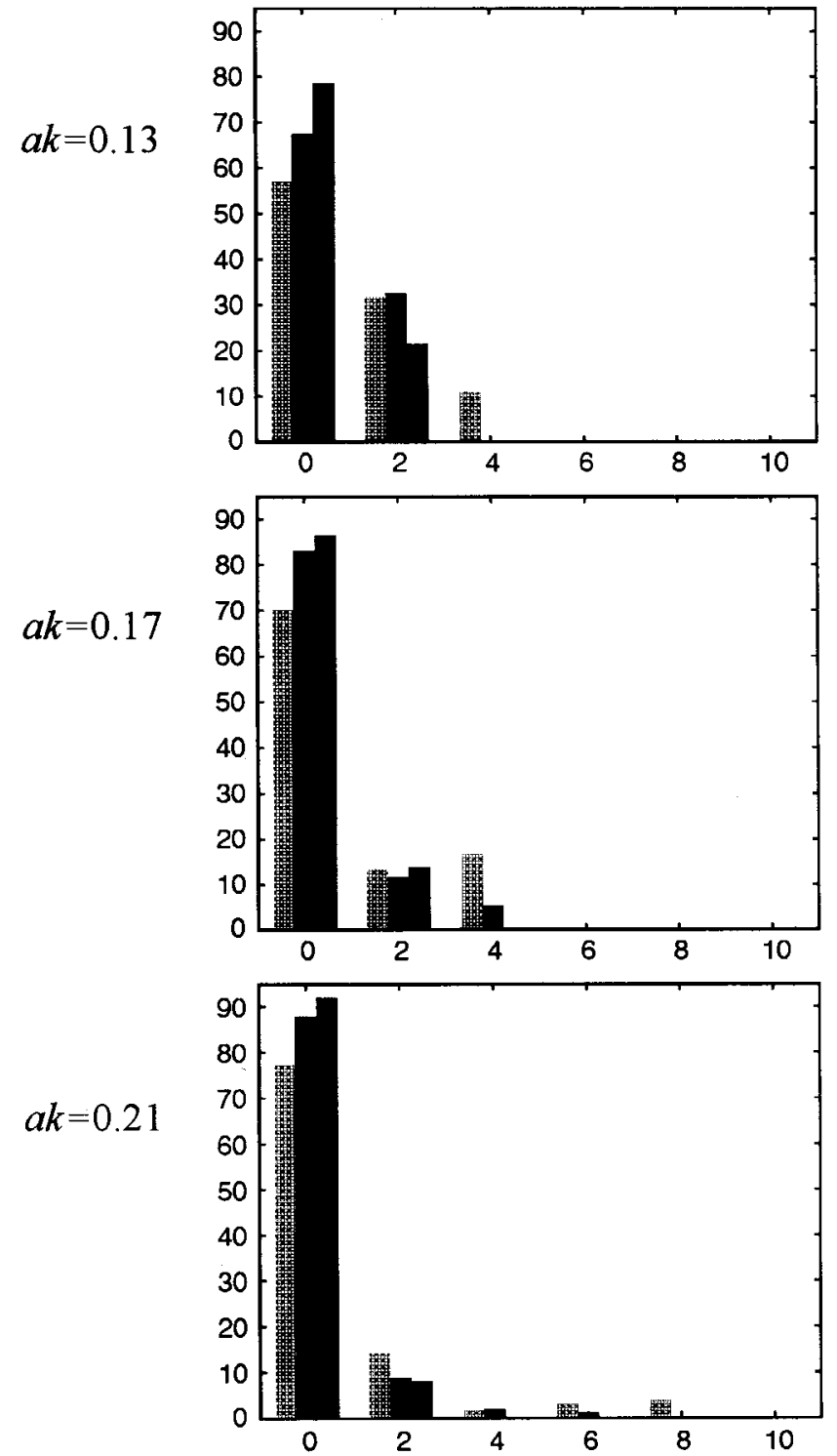

Fig. 10. Percentage of time (vertical axis), during which the specified number of grown satellites (horizontal axis) is present in the non-conservative system. All the parameters of the system (including the amount of forcing and dissipation) are the same as in Fig. 4, but three different values of the basic wave steepness are used. Evolution was traced up to 10000 time units (inverse values of the basic wave frequency). A satellite was considered grown if its energy exceeded a certain level relative to the total energy of the system: $2 \%$ (light bars), $4 \%$ (medium bars), $6 \%$ (dark bars). formation of horse-shoe patterns that can be revealed by an analysis of the simplest low-modal systems. For this purpose, we first consider some basic facts referring to the class II instability in a conservative system.

A monochromatic wave of amplitude $a$ and wavevector $\mathbf{k}_{a}=(1,0)$ is known to be unstable with respect to the pairs of oblique satellites with wavevectors $\mathbf{k}_{b}, \mathbf{k}_{c}$ satisfying (8), if a certain condition on $p, q$ implied by (9) is fulfilled. Within the framework of the Zakharov equation, the corresponding condition can be easily obtained in an explicit form. Indeed, linearizing (10) and considering the satellites as perturbations, a pair of decoupled linear equations for each pair readily follows

$$
\begin{aligned}
& \mathrm{i} \hat{b}_{t}=W_{b c a a a} \bar{a}^{3} \hat{c}^{*} \exp (-\mathrm{i} \sigma t)+\gamma_{b} \hat{b}, \\
& \mathrm{i} \hat{c}_{t}=W_{b c a a a} \bar{a}^{3} \hat{b}^{*} \exp (-\mathrm{i} \sigma t)+\gamma_{c} \hat{c},
\end{aligned}
$$

where

$$
\begin{aligned}
& \sigma=3 \omega_{a}-\omega_{b}-\omega_{c}+\left(3 V_{a a a a}-2 V_{a b a b}-2 V_{a c a c}\right)|\bar{a}|^{2}, \\
& \bar{a}=a(0), \quad \hat{b}=b \exp \left(-\mathrm{i}\left[\omega_{b}-2 V_{a b a b} \bar{a}^{2}\right] t\right), \\
& \hat{c}=c \exp \left(-\mathrm{i}\left[\omega_{c}-2 V_{a c a c} \bar{a}^{2}\right] t\right) .
\end{aligned}
$$

Condition of instability has the form

$$
\begin{array}{r}
\operatorname{Re}\left(\sqrt{4 W_{b c a a a}^{2}|\bar{a}|^{6}-\left(\sigma-\mathrm{i}\left(\gamma_{b}-\gamma_{c}\right)\right)^{2}}\right)> \\
-\left(\gamma_{b}+\gamma_{c}\right) .
\end{array}
$$

An example of the instability domain for $\gamma_{b}=\gamma_{c}=$ $-5 \cdot 10^{-4}$ (the standard value for our simulations) and wave steepness 0.21 is drawn in Fig. 11. For comparison, the instability domain for purely conservative case is also given (Fig. 12). According to such a linearized theory, all the initially small oblique satellites in the instability domain grow exponentially, with the rate

$$
\operatorname{Re}\left(\sqrt{4 W_{b c a a a}^{2}|\bar{a}|^{6}-\left(\sigma-\mathrm{i}\left(\gamma_{b}-\gamma_{c}\right)\right)^{2}}\right)+\gamma_{b}+\gamma_{c} .
$$

Above a certain threshold of satellites' amplitude, still much smaller than the amplitude of the basic wave, their evolution becomes nonlinear and different pairs begin to interact. If only one pair is present, its evolution is governed by full set (11); the satellites grow as long as sine of the phase is negative, pass the maximum of amplitude corresponding to zero phase and then decay. The process is recurrent, linearized system (18) being valid as an approximation on each cycle for the exponential 'tails' in the neighbourhood of the point of smallest amplitude of the satellites. A complete analytical solution to (11) is available but rather bulky. Analysis of the most interesting case in the present context, when the initial amplitude of the satellites is close to zero and can be considered as a small parameter, is performed in the Appendix.

The presence of just one extra pair makes the problem much more difficult. The equations are not integrable any more and even the construction of an asymptotic solution is not straightforward. However, as noted 


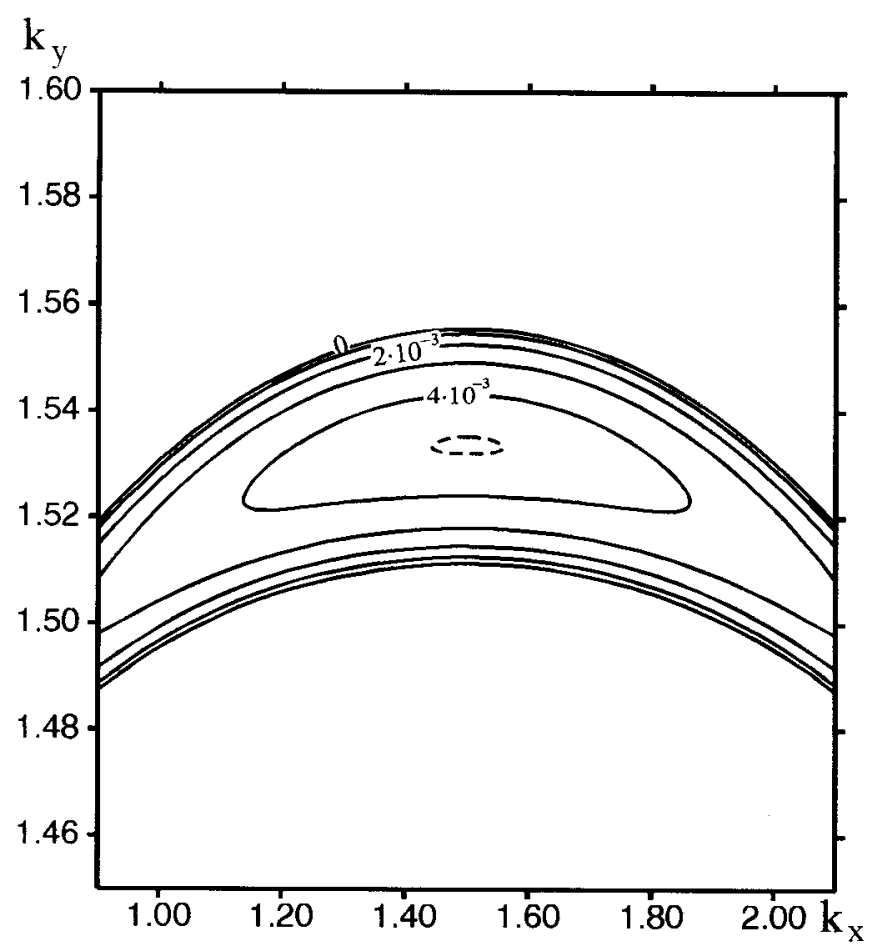

Fig. 11. Instability domain in k-plane for system (18). Growth rates for initial steepness of the basic wave 0.21 are shown. Small dissipation for the satellites is included $\left(\gamma_{b}=\gamma_{c}=-5 \cdot 10^{-4}\right)$.

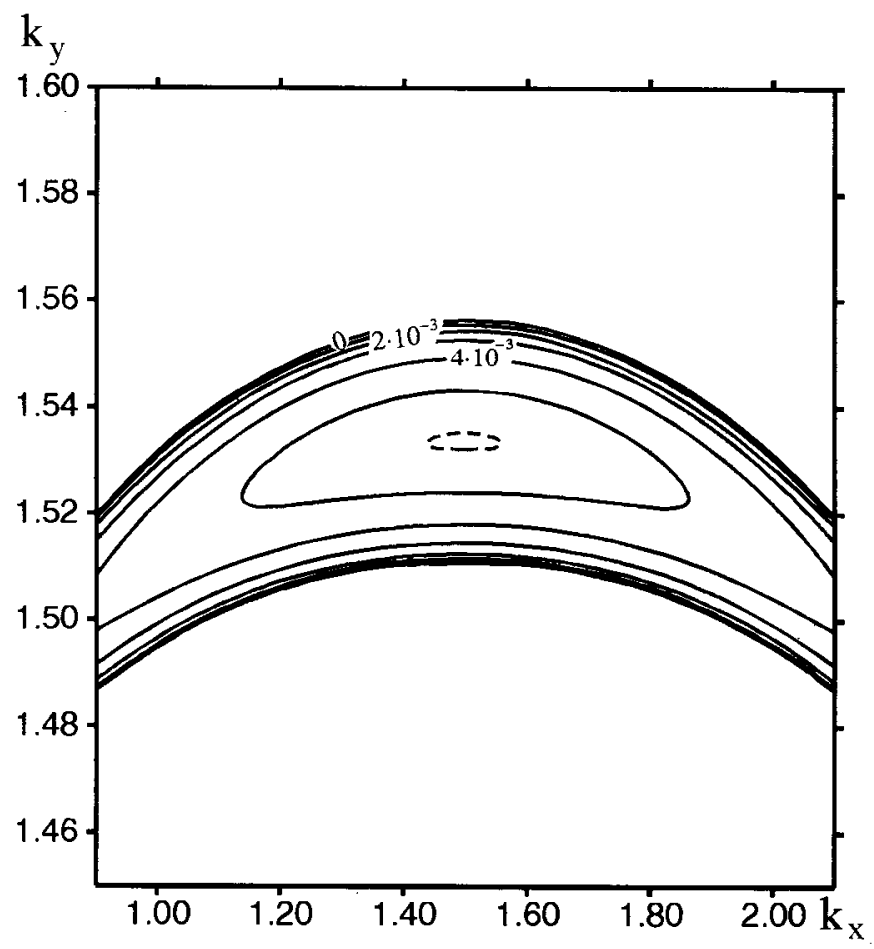

Fig. 12. Instability domain in k-plane for system (18), with $\gamma_{b}=\gamma_{c}=0$, for initial steepness 0.21 . above, the solution to system (16) obtained numerically typically shows a relatively simple scenario of evolution (Figs. 6, 7) that suggests to consider the problem asymptotically as, in the zeroth order, an alternation of two three-wave systems. In particular, at the initial stage of the evolution linear regime is realized. Then, at the moment when the nonlinear effects of interaction between the satellites become significant, selection of modes occurs, so that one pair of satellites continues to grow, while the other one slowly decays. We emphasize that this selection of pairs occurs at the level of satellite amplitudes much smaller than the amplitude of the basic wave. This relative smallness of the satellite amplitude at this crucial stage will be utilized as the small parameter for the construction of an asymptotic description of the selection process. The subsequent growth of the selected pair approximately follows the evolution of a three-wave system, since the influence of the other pair on the system dynamics at this stage is negligible, to a leading order. After passing the maximum of modulation, the amplitudes of both pairs again become comparable, for another selection, and then the process approximately repeats.

In principle, an approximate solution to system (16) can be obtained in this way by constructing the asymptotic solution for each stage of the evolution and matching the resulting expansions. Since our primary interest lies in the selection process, consider (16) for the stage $B \simeq D \ll A$ with the nonlinear interactions among the satellites taken into account.

First, it is helpful to make the scaling involved more explicit. The Zakharov equation (16), derived as a result of expansion in powers of wave steepness assumed to be $O(\varepsilon)$, is nevertheless written in the traditional normalization where the amplitude of the basic wave becomes $O(1)$, while the time scale is linked to the order $W_{\text {bcaaa }} \simeq W_{\text {deaaa }} \simeq O\left(\varepsilon^{3}\right)$. It makes sense to assume that the two pairs lie close to the point of the maximum linear growth rate in $\mathbf{k}$-plane, so that one may put $W_{\text {bcaaa }} \cong W_{\text {deaaa }} \equiv W$. All the other coefficients (namely, $\delta_{j}, P_{j}, M_{j}, N_{j}$, where $j=1,2$ ) are of the or$\operatorname{der} \varepsilon^{2}$, i.e., one order of magnitude larger. However, in virtue of condition (19), the total initial frequency mismatch with the nonlinear frequency shifts caused by the basic wave, $\delta_{j}+P_{j} A(0)^{2}$ is of the order of the largest term due to the quintet interaction, i.e., $O\left(\varepsilon^{3}\right)$. Introducing $\tau=W t$ as a slow timescale, the typical ratio of amplitudes of satellites and the fundamental at the point where selection occurs $B / A \simeq D / A=\mu$ as a new small parameter and $\hat{M}_{j}=\varepsilon M_{j} / W, \hat{N}_{j}=\varepsilon N_{j} / W$, $\hat{P}_{j}=\varepsilon P_{j} / W$ as new $O(1)$ coefficients, we can now explicitly present the set (16) in terms of two small parameters $\mu$ and $\varepsilon$ retaining only the leading order terms in $\mu^{2}$ :

$$
\begin{aligned}
& A_{\tau}=3 A^{2} B^{2} \sin \Phi_{1}+3 A^{2} D^{2} \sin \Phi_{2}+\hat{\Gamma}_{a} A \\
& B_{\tau}=-A^{3} B \sin \Phi_{1}+\hat{\Gamma}_{b} B
\end{aligned}
$$




$$
\begin{aligned}
D_{\tau}= & -A^{3} D \sin \Phi_{2}+\hat{\Gamma}_{d} D \\
\Phi_{1 \tau}= & \frac{\left[\delta_{1}+P_{1} A_{0}^{2}\right]}{W}+\frac{\mu^{2} \hat{P}_{1}\left(A^{2}-A_{0}{ }^{2}\right)}{\varepsilon} \\
& +\frac{\mu^{2} \hat{N}_{1} D^{2}}{\varepsilon}-2 A^{3} \cos \Phi_{1}, \\
\Phi_{2 \tau}= & \frac{\left[\delta_{2}+P_{2} A_{0}{ }^{2}\right]}{W}+\frac{\mu^{2} \hat{P}_{2}\left(A^{2}-A_{0}{ }^{2}\right)}{\varepsilon} \\
& +\frac{\mu^{2} \hat{N}_{2} B^{2}}{\varepsilon}-2 A^{3} \cos \Phi_{2},
\end{aligned}
$$

where $A_{0}=A(0), \hat{\Gamma}=\Gamma / W$.

System (20) enables one to understand the mechanism of the selection process. At the initial stage of evolution, when $B^{2} / A^{2} \ll \mu$ and $D^{2} / A^{2} \ll \mu$, the linear regime is realized: both pairs of satellites grow, provided that $\Phi_{1}$ and $\Phi_{2}$ are both negative. When the satellites attain a certain threshold amplitude, the second terms in the right-hand sides of the phase equations in (20) become of order $O(1)$. The signs of these frequency-shifting terms due to the presence of other pair of satellites are such that each pair tends to push the phase of the other pair from the value which is favourable for the growth of amplitude, until one of the phases starts to rotate, preventing the subsequent growth of the corresponding pair. Afterwards, only one of the pairs grows, while the other one, with the rapidly rotating phase, is decaying. The presence of the small parameter in the denominator of the frequency-shifting terms specifies the characteristic threshold level of the satellite amplitude where the selection occurs: $B \simeq D \simeq \sqrt{\varepsilon} A$. One can describe this mechanism as the 'rivalry' between the pairs, so that each one, while growing, pushes the other one out of the resonance with the fundamental via the frequency shift.

We already mentioned the interesting effect exhibited by the simulations: the maximal amplitude of the remaining pair noticeably exceeds that of the same pair in the isolated three-wave system. In other words, the presence of the other pair, despite the fact that it remains small, acts like a catalyst enhancing the energy exchange between the growing satellite and the fundamental. This fact can be easily explained by comparison of (20) with the single-pair system. For example, assuming that the first pair is selected for growth and the second one damped, the system for the first pair in the absence of the second one has the form of the set (13). For the near-resonance $\delta_{1}+P_{1} A_{0}^{2}=O\left(\varepsilon^{3}\right)$, while the two constituents in this sum are an order of magnitude larger and therefore should have opposite signs, $\delta_{1}>0, P_{1}<0$. The growth of $B$ in (13) is thus controlled by the change of the amplitude of the fundamental: when $A$ diminishes, the variation of the frequency shift $P_{1}\left(A^{2}-A_{0}^{2}\right) / W$ becomes large and positive, leading to counterclockwise rotation of phase. Meanwhile, in the corresponding phase equation in (20) $N_{1}<0$, so that the term $N_{1} D^{2}$ is always negative, and thus tends to compensate the effect of the frequency shift for this pair, while the similar term in the other phase equation drives the phase of the second pair out of equilibrium. Hence, the effect tends to stabilize the phase of the first one at the value that is favourable for growth, eventually resulting in the increase of the maximal amplitude.

In order to build a quantitative model of the selection process, one needs to integrate (20). Though this is not possible in general case, there is an integrable class of situations of interest corresponding to the case which we refer to as of strong dissipation: the damping nearly compensates the maximal growth rate due to the conservative class II instability but does not exceed it, i.e., the resulting actual growth rate is much smaller than $O\left(\Gamma_{b, d}\right)$ or $O(W)^{1}$. Then the growth rate of the amplitudes is an order of magnitude smaller than the typical rate of change for the phases, enabling us to obtain the explicit solution for the phases in the form

$\sin \Phi_{1}=$

$$
2 \frac{\sqrt{-R_{1} R_{2}} \sinh \left[\sqrt{-R_{1} R_{2}}(t+C)\right]}{R_{1}+R_{2}-\left(R_{1}-R_{2}\right) \cosh \left[\sqrt{-R_{1} R_{2}}(t+C)\right]},
$$

$R_{1}=\delta_{1}+P_{1} A^{2}+N_{1} D^{2}-W A\left(9 B^{2}-2 A^{2}\right)$,

$R_{2}=\delta_{1}+P_{1} A^{2}+N_{1} D^{2}+W A\left(9 B^{2}-2 A^{2}\right)$,

where $C$ is a constant determined from the initial condition for the phase. The slow time dependence enters through the amplitudes $A, B$ and $D$. According to Eq. (21), the behaviour of $\sin \Phi_{1}$ depends on the signs of $R_{1}$ and $R_{2}$. If

$\left|\delta_{1}+P_{1} A^{2}+N_{1} D^{2}\right|<W A\left|9 B^{2}-2 A^{2}\right|$,

then $R_{1} R_{2}$ is negative, and $\sin \Phi_{1}$ is a hyperbolic function of time; in the opposite case, $\sin \Phi_{1}$ oscillates; when $R_{1} R_{2}=0, \Phi_{1}$ passes through zero. Taking into account the signs of the coefficients, we obtain from (22)

$$
\begin{aligned}
\frac{\delta_{1}+P_{1} A^{2}-}{W A\left(2 A^{2}-9 B^{2}\right)}<D^{2}< \\
\frac{-N_{1}}{\frac{\delta_{1}+P_{1} A^{2}+W A\left(2 A^{2}-9 B^{2}\right)}{-N_{1}}},
\end{aligned}
$$

and, in the same way, the condition for $\Phi_{2}$,

$$
\begin{aligned}
\frac{\delta_{2}+P_{2} A^{2}-}{W A\left(2 A^{2}-9 D^{2}\right)}<B^{2}< \\
\frac{-N_{2}}{\delta_{2}+P_{2} A^{2}+W A\left(2 A^{2}-9 D^{2}\right)} \\
-N_{2}
\end{aligned}
$$

Inequalities (23), (24) constitute the necessary condition for the growth of $B$ and $D$ respectively. Consider, for instance, (23). At the initial moment, the left boundary of the interval is negative, provided the pair of satellites with the amplitude $B$ lies in the instability domain (cf. (19)), while the right boundary is positive. Since initially $D$ is close to zero, inequality (23) is satisfied. The

\footnotetext{
${ }^{1}$ Note that this regime still does not violate the Hamiltonian structure to a leading order.
} 
same conclusion holds for another pair. Consequently, for a certain period of time $\Phi_{1}$ and $\Phi_{2}$ both remain in the lower half-plane; this means the simultaneous growth of $B$ and $D$. If, in this process, one of the right inequalities in (23), (24) breaks, sine of $\Phi_{1}$ or $\Phi_{2}$, respectively, starts to oscillate, and the further growth of the corresponding pair of satellites is suppressed. On the other hand, breaking of the left inequality in (23) or (24) corresponds to the limit of the amplitude growth. For instance, provided that the right inequality in (23) holds, the amplitude $B$ grows as long as

$$
\frac{\delta_{1}+P_{1} A^{2}-W A\left(2 A^{2}-9 B^{2}\right)}{-N_{1}}<D^{2},
$$

so that, as noted above, the presence of small $D$ enlarges the maximal amplitude for $B$.

If $\delta_{1}+P_{1} A_{0}^{2}$ and $\delta_{2}+P_{2} A_{0}^{2}$ are both large, neither $B$ nor $D$ can break the right inequality in (23), (24). This is a rather exceptional case, possible only near the boundary of the instability domain with the smallest $q$. Then, the selection mechanism does not work, but instead, the 'mutual instability enhancement' scenario (cf. Fig. 8) is realized; despite the fact that the linear instability rate is small, both pairs eventually grow to considerable amplitudes.

Solution (21) stands for the model of the selection proccss for the case when dissipation is strong, in the sense specified above. The case of smaller dissipation is not tractable analytically; however, numerical experiments demonstrate that the above description of the selection mechanism remains qualitatively valid.

Thus, out of two pairs of satellites present in the system, normally only one pair grows to considerable amplitude, according to the described mechanism. Certainly, it would be important to have the possibility to determine a priori, in each case, which pair out of two given ones is selected. A complete answer to this question is beyond the scope of the present work; however, it is possible to list some of the factors acting in favour of a given pair. In particular, a pair of satellites has more chances to be selected if:

(a) the initial value of its phase is close to $-\pi / 2$,

(b) the pair lies close to the point of maximal linear growth rate,

(c) coefficient $N_{j}$ is small,

(d) nonlinear frequency mismatch is initially positive.

In general, these factors are contradictory; for instance, (b) tends to favour symmetric pairs (with $p=0$, see (8)), while (c) sets preference for $p$ close to $\frac{1}{2}$, etc. Numerical experiments show in most cases the more or less clear preference for the symmetric pairs in the vicinity of the maximal linear instability point; the same seems to be demonstrated by observations. However, in wave tank experiments, where the transverse length scale is prescribed by the tank width, the situation might be difierent.

Turning back to our main subject, we can now describe the process of the formation of horse-shoe patterns. Although at the initial stage of instability development a large number of pairs of satellites grows, each of the pairs tends to destabilize the phases of all the other pairs and thus to suppress their further growth, so that eventually only a few pairs of satellites (in most cases, just one pair) survive and can attain considerable amplitudes. In course of the development of the instability, the phases of the growing pairs are negative, typically close to $-\pi / 2$, corresponding to the existence on the free surface of downwind oriented horse-shoe patterns, while the phases of all the other pairs rapidly change. Soon after passing the maximum of modulation, for a short period of time the phases of the grown satellites are inverted, but then a number of other satellites starts to develop, forcing the phases of the decaying waves to rotate. After that, the nonlinear mechanism selects a new pair for growth, and the process is reiterated.

\section{Effects due to the finite width of the spec- trum of the basic wave}

So far, we have based our analysis on the assumption that the wave field consists of a finite set of monochromatic waves represented by a number of $\delta$-pulses in wavevector space [Cf. (6)]. It is obvious, however, that an actual wave field is continuous, so that the consideration of the process of interaction of monochromatic waves should be regarded as an approximation to the interaction of finite bandwidth wavepackets. In particular, the spectrum bandwidth of the basic wave, generated either by wind or by wavemaker, cannot be less than $\varepsilon$. A detailed study of the effects due to the finite bandwidth of this spectrum represents a separate problem which would deserve a special study. The most important effect in this respect is clearly the presence of the modulational (Benjamin-Feir) instability of the fundamental wavepacket. Does the presence of much faster $\left(O(\varepsilon)^{-2}\right)$ and more energetic four-wave (class I) processes preserve intact the evolution scenaria established above?

As a first approximation, the effect of the modulational instability can be modeled just by adding of one or more pairs of additional initially small satellites $\mathbf{k}_{j \pm}=$ $\left(1 \pm \Delta_{j}, 0\right), j=1,2, \ldots$, where $\mathbf{k}_{0}=(1,0)$ is the basic wave central wavevector, $\Delta_{j}$ lie within the domain of the Benjamin-Feir instability (see, e.g. Craik, 1985).

Based on numerous simulations in wide range of the parameters involved (two samples are given in Figs. 13, 14) we conclude that qualitatively, the account of the finite bandwidth of the basic wave does not alter the already established scenaria of field evolution on $\varepsilon^{-3}$. 


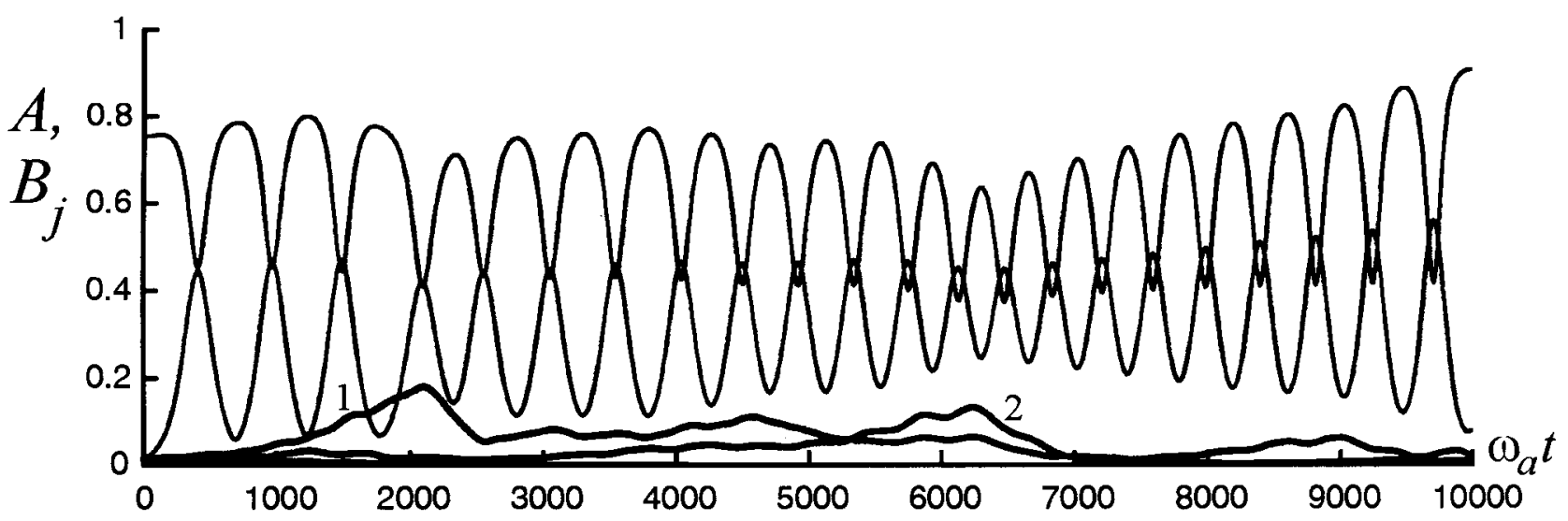

$\sin \Phi$
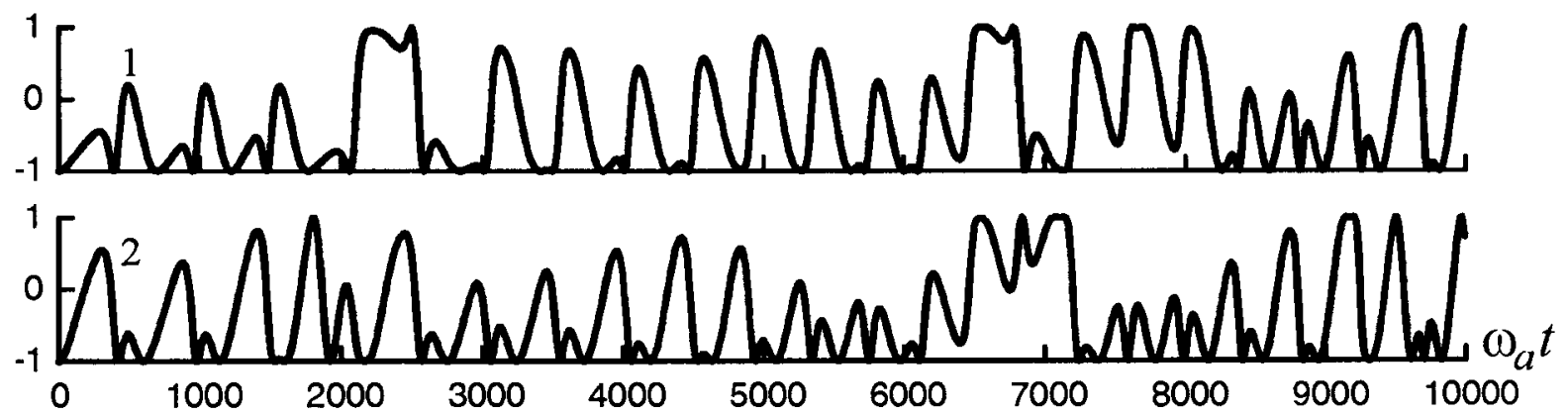

Fig. 13. Evolution of the system of 87 waves obtained by the numerical solution of the Zakharov equation perturbed by small nonconservative effects. The system consists of the basic wave, with initial amplitude $A(0)=0.755$ (corresponding to steepness 0.17 ), 42 pairs of initially small $\left(B_{j}(0)=0.014, j=1,2, \ldots, 84\right)$ five-wave satellites (the same set was used in Sect. 3, Figs. 3,4), and one pair of four-wave (Benjamin-Feir) satellites $\left(\mathbf{k}_{j}=(1 \pm 0.27,0)\right)$, with the same initial amplitude. Weak forcing for the basic wave and weak dissipation for five-wave satellites $\left(\Gamma_{0}=5 \cdot 10^{-5} \omega_{0}, \Gamma_{j}=-5 \cdot 10^{-4} \omega_{j}, j=1,2, \ldots, 84\right)$ are included. For two most unstable five-wave pairs (plotted with bold curves and numbered 1,2 in the amplitude plot), evolution of phase is shown.

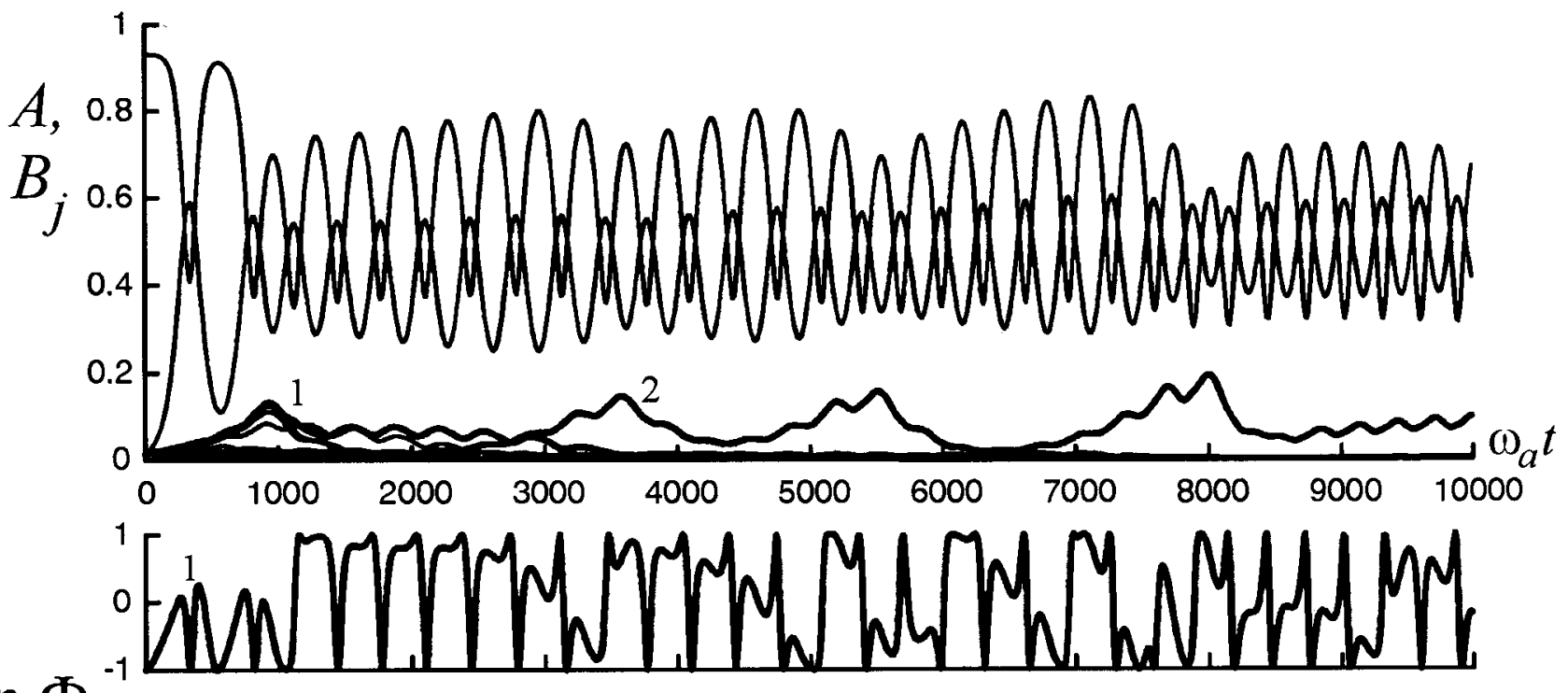

$\sin \Phi$

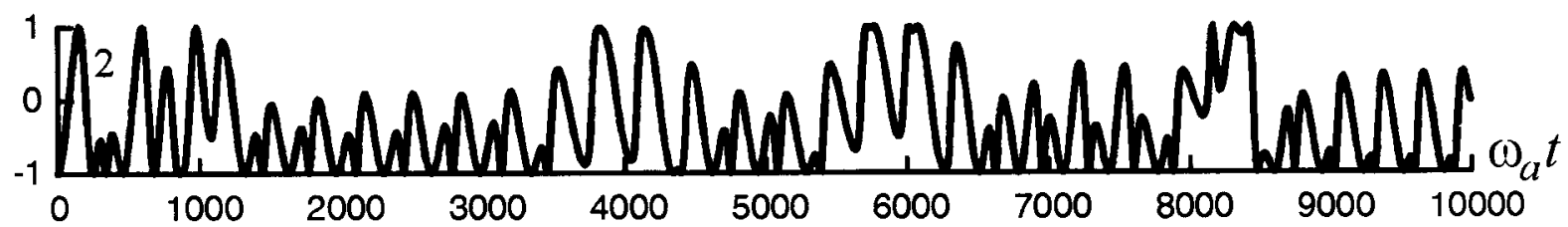

Fig. 14. As in Fig. 13, for larger initial steepness of the basic wave $(A(0)=0.933$, corresponding to steepness 0.21 ). 


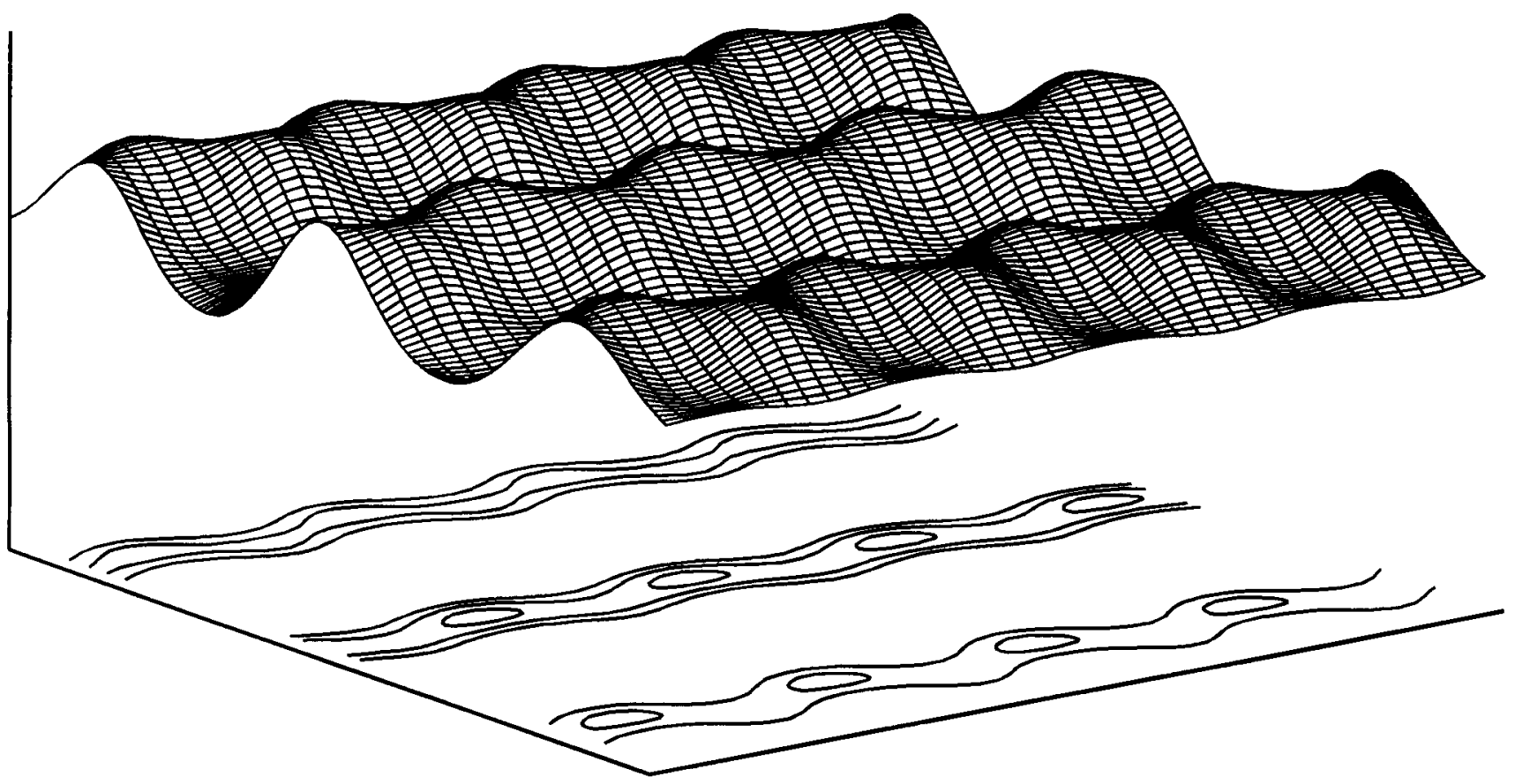

Fig. 15. Plot of the free surface for the wave field evolution shown in Fig. 13, for $t=1300 \omega_{0}^{-1}$. Shape of the wave crests is visualized with a few contour lines beneath the surface.

timescale, although quantitatively the effect could be quite noticeable (Cf. Figs. 4 and 13, 5 and 14, respectively). To illustrate this point further, instantaneous plots of the free surface of the wave field evolution for the examples plotted in Fig. 10 are shown in Figs. 15, 16 , where both two-dimensional modulations and threedimensional horse-shoe patterns are present.

It has been previously suggested by $\mathrm{Su}$ and Green (1984) that the class I processes, which have shorter characteristic time of development, may trigger the class II instability. Our results do not support this hypothesis. Moreover, in accordance with the earlier simulations of Stiassnie and Shemer (1987), the presence of the class I instability imposing fast oscillations to the amplitudes and phases of the fundamental and the class II satellites appears to inhibit the five-wave processes. The large number of runs of the numerical model for various values of the basic wave steepness and dissipation/generation ratio supported this conclusion. Qualitatively this fact can be easily explained, averaging, by virtue of the existing separation of scales, the equations over fast Benjamin-Feir oscillations: the 'averaged phase' of the satellite will always differ from the optimal one $-\pi / 2$. The more intense is the fast modulation, the larger is the deviation from the optimal value and, thus, the less are the growth rates. (In the absence of fast oscillations the phase of the growing satellites is automatically set at the optimal value.) However, in order to specify quantitatively how the account of Benjamin-. Feir modulation inhibits the class II processes, a special study is necessary which is not in the scope of the present work.

\section{Discussion}

We will now summarize the main results of the paper and discuss their implications and the approximations involved.

Numerical and analytical consideration of a gravity wave instability with respect to four- and five-wave processes has enabled us to establish a number of properties referring to the formation of three-dimensional patterns on the free surface.

We have shown that nonlinear evolution of five-wave instability in the generic case can be adequately represented as a low-dimensional process. Though at a linear stage all linearly unstable harmonics do grow simultaneously, most of them cannot attain considerable amplitudes. Instead, at a nonlinear stage the subsequent growth is arrested by the selection mechanism: nonlinear frequency shifts lead in most cases to the competition among the satellites, so that they tend to push each other out of the resonance zone. Since this zone is quite narrow, eventually only a small number (typically, a single pair) of satellites survives and remains the prevailing one, at least during one cycle of the satellite growth and decay (modulation cycle):

Existence of this mechanism leads to several important consequences. First, the developed class II instability remains essentially low-modal, at least at characteristic times of the order of modulation cycle. This greatly simplifies the theoretical study of the five-wave decay of a basic wave, being also in agreement with observations (Collard and Caulliez, 1999). Second, the harmonics pushed out by a growing satellite at some value of their 


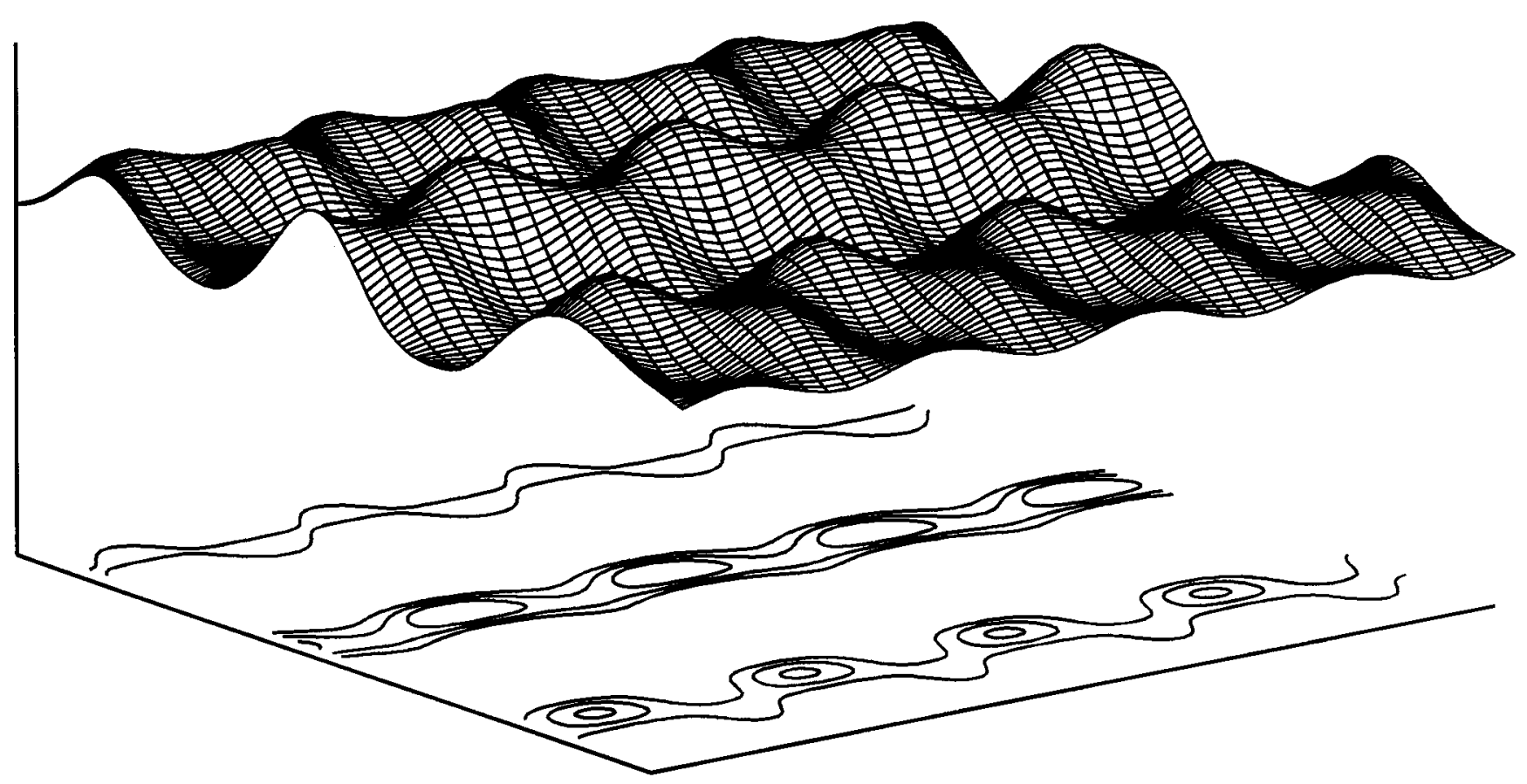

Fig. 16. Plot of the free surface for the wave field evolution shown in Fig. 13, for $t=2000 \omega_{0}^{-1}$.

amplitude (that can be easily estimated) take part in the formation of noise, thus allowing one to introduce the concept of natural noise level. Third, the mechanism controls the effective phase of a growing satellite in such a way that it remains negative for growing and grown satellites and is indeterminate (rapidly changing) for decaying ones. This gives a scenario for the emergence of horse-shoe patterns on the water surface: at each moment a certain pair of harmonics grows with a fixed phase corresponding to downwind orientation of the patterns, while the phases of all the other satellites are rapidly rotating. According to this scenario, the formation of patterns occurs rather rapidly, with the characteristic time of the class II instability, and each particular 'individual modulation' exists for a about the same characteristic time period. This correctly describes the character of the observed sporadic patterns. Moreover, the scenario explains the long standing enigmatic fact of horse-shoe pattern observations even in tanks without wind: since the size of the installations is finite, just a fraction of the first modulation cycle is typically available. Furthermore, in full agreement with the tank observations, the mechanism works only for the steepness of the basic wave exceeding a certain level. Such a threshold character of the process is clearly attributed to the rate of dissipation: since the inviscid growth rate of the class II instability is proportional to $\varepsilon^{3}$, too small steepness of the fundamental is unable to provide the growth rate sufficient to overcome the dissipation. It is important to note, however, that apart from this threshold, no essential qualitative dependence of dynamics on the amplitude of the basic wave was noticed.
The role of dissipation is more diverse. It creates the important phase shift of grown modes, making the phases at the maxima of the satellites amplitude negative, and, besides that, enhances the phase dynamics asymmetry by prolonging the growth and shortening the decay of satellites. However, it is important to note that steep gravity waves under the action of wind are likely to develop breakers which obviously require much more sophisticated description of nonlinear non-conservative mechanisms compared to the extremely simple generation/dissipation model used in this work. Account of nonlinear non-conservative mechanisms (yet to be elaborated) will certainly enrich the dynamics. Nevertheless, we expect that the basic physics established here will remain qualitatively intact.

Thus, the simple model considered gives the qualitative description of the sporadic horse-shoe phenomenon which is consistent with the available observations.

The model seems to be especially adequate for laboratory wind-wave experiments, where wavemaker produces a single almost monochromatic wave and other external factors are excluded, so that the wave propagates in nearly homogeneous low-level noise. In natural conditions, however, such a quasimonochromatic representation is possible for swell only, while wind waves always have a certain spectral bandwidth. When the bandwidth is of the order of wave steepness $\varepsilon$, the presence of stronger and faster Benjamin-Feir modulations of the basic wave should be taken into account for the consideration of slower dynamics due to quintet interactions. Although we have demonstrated the principal fact of the absence of qualitative changes to the considered 
three-dimensional processes, the detailed consideration of the influence of quartet interactions was not in the scope of the present work. Still, it should be noted that our study confirmed that in general Benjamin-Feir modulations tend to inhibit the class II instability, so that one cannot rule out the possibility that this instability may disappear under certain conditions. This question, as well as the question related to the case when the basic wave spectrum is much wider than $\varepsilon$, is open and definitely merits a special study.

Among the implicitly adopted assumptions, we presumed the initial wave field to be spatially homogeneous, so that an adequate description of the evolution could be given solely in terms of purely temporal processes. The possible role of spatial variations remains an open question.

Another limitation of the present study is the unavoidable reduction of a system consisting of a basic wave and a noise of continuous spectrum to that of a certain (although large) number of harmonics. One cannot exclude the possibility that a system with the infinite number of degrees of freedom could manifest qualitatively different behaviour. The evolution may also depend on the statistics of the initial ensemble (which is, in fact, unknown). A number of model runs with Gaussian statistics for initial amplitudes and phases of harmonics has shown the clear tendency for selection, in accordance with the conclusions of the present work. No qualitative changes in the system behaviour were noted while varying the number of random satellites. Still, this number in the present study had the upper limit of about 200-250 set by the computer capacity, so some caution is needed in addressing the behaviour of infinitely dimensional continuous systems.

Having demonstrated the low-modal character of the dynamics of the complex system considered, the present work actually suggests that its evolution can be treated, with a good accuracy, as a sequence of three-wave systems with random choice of the satellite pair (where the corresponding statistics is specified by the unknown yet statistics of the background noise), this process describing the formation of sporadic horse-shoe patterns. The three-wave system in itself can exhibit a variety of dynamical regimes, the outcome of which depends on the specific combinations of the system parameters and initial conditions. In particular, as it was shown by SBK, such a three-mode system is capable to evolve to attractors corresponding to permanent horse-shoes, while the further work (Badulin and Shrira, 1999) demonstrated the existence of more complicated attractors corresponding to oscillating patterns and showed that the characteristic times of evolution towards these attractors can be sufficiently small. However, for a quantitative description of the evolution in statistical terms, a more precise account of non-conservative effects in the model and a deeper understanding of the properties of such a model are needed.

\section{Appendix Quintet interactions within a three- wave system}

Consider the interaction of three quasimonochromatic wavepackets with amplitudes $a, b, c$ and wavevectors $\mathbf{k}_{a}, \mathbf{k}_{b}, \mathbf{k}_{c}$ that satisfy the relation (7). Dynamics of such a triad is governed by the system (Cf. (10))

$$
\begin{aligned}
a_{t} & =-\mathrm{i} \Omega_{a} a-3 \mathrm{i} W b c\left(a^{2}\right)^{*}, \\
b_{t} & =-\mathrm{i} \Omega_{b} b-3 \mathrm{i} W c^{*} a^{3} \\
c_{t} & =-\mathrm{i} \Omega_{c} c-3 \mathrm{i} W b^{*} a^{3}
\end{aligned}
$$

where

$$
\begin{aligned}
& \Omega_{a}=\omega_{a}+V_{a a a a}|a|^{2}+2 V_{a b a b}|b|^{2}+2 V_{a c a c}|c|^{2}, \\
& \Omega_{b}=\omega_{b}+2 V_{a b a b}|a|^{2}+V_{b b b b}|b|^{2}+2 V_{b c b c}|c|^{2}, \\
& \Omega_{c}=\omega_{c}+2 V_{a c a c}|a|^{2}+2 V_{b c b c}|b|^{2}+V_{c c c c}|c|^{2},
\end{aligned}
$$

other notation is as in Sect. 3. System (A1) is Hamiltonian and integrable, with the Hamiltonian function in the form

$$
\begin{aligned}
H= & \omega_{a}|a|^{2}+\omega_{b}|b|^{2}+\omega_{c}|c|^{2} \\
& +\frac{1}{2}\left(V_{a a a a}|a|^{4}+V_{b b b b}|b|^{4}+V_{c c c c}|c|^{4}\right) \\
& +2\left(V_{a b a b}|a|^{2}|b|^{2}+V_{a c a c}|a|^{2}|c|^{2}+V_{b c b c}|b|^{2}|c|^{2}\right) \\
& +W\left(a^{3}\right)^{*} b c
\end{aligned}
$$

and momentum conservation laws

$$
\begin{aligned}
|a|^{2}+\frac{3}{2}\left(|b|^{2}+|c|^{2}\right) & =I_{1} \equiv \text { const } \\
\frac{3}{2}\left(|b|^{2}-|c|^{2}\right) & =I_{2} \equiv \text { const },
\end{aligned}
$$

Following the lines of Shemer and Stiassnie (1985), we rewrite system (A1) in the form

$$
\begin{aligned}
\left(a a^{*}\right)_{t} & =6 W \operatorname{Im}\left[\left(a^{3}\right)^{*} b c\right], \\
\left(b b^{*}\right)_{t} & =-2 W \operatorname{Im}\left[\left(a^{3}\right)^{*} b c\right], \\
\left(c c^{*}\right)_{t} & =-2 W \operatorname{Im}\left[\left(a^{3}\right)^{*} b c\right] .
\end{aligned}
$$

and define formally a real function $Z$ by the relation

$\frac{\mathrm{d} Z}{\mathrm{~d} t}=\operatorname{Im}\left[\left(a^{3}\right)^{*} b c\right]$,

so that, after integration,

$$
\begin{aligned}
& |a|^{2}=6 W Z+\left|a_{0}\right|^{2}, \\
& |b|^{2}=-2 W Z+\left|b_{0}\right|^{2} \\
& |c|^{2}=-2 W Z+\left|c_{0}\right|^{2},
\end{aligned}
$$

and it makes sense to assume that at the initial moment $(t=0) Z$ is zero, so that the constants $a_{0}, b_{0}, c_{0}$ are the initial values of the corresponding amplitudes.

It is easy to see from (A1) that

$$
\operatorname{Re}\left[\left(a^{3}\right)^{*} b c\right]=-\int_{0}^{Z} \Phi \mathrm{d} Z+\operatorname{Re}\left[\left(a_{0}^{3}\right)^{*} b_{0} c_{0}\right]
$$




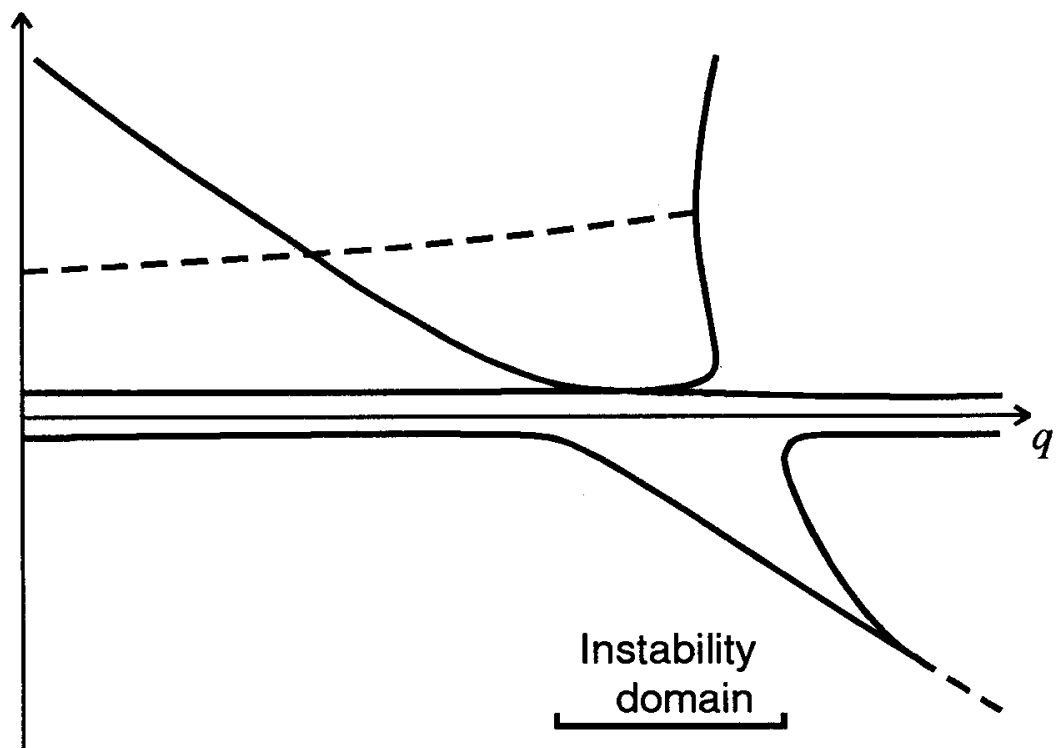

Fig. A1. Schematic plot of the structure of roots of the polynomial $\mathcal{P}_{5}$ for the three-wave system $\mathbf{k}_{a}=(1,0)$, $\mathbf{k}_{b}=(3 / 2, q), \mathbf{k}_{c}=(3 / 2,-q)$. Dependence of the roots on $q$ is shown. For complex roots, real parts are plotted (dashed curves).

and thus

$$
\begin{aligned}
\left(\frac{\mathrm{d} Z}{\mathrm{~d} t}\right)^{2} & =|a|^{6}|b|^{2}|c|^{2} \\
& -\left\{-\int_{0}^{Z} \Phi \mathrm{d} Z+\operatorname{Re}\left[\left(a_{0}^{3}\right)^{*} b_{0} c_{0}\right]\right\}^{2}
\end{aligned}
$$

We have obtained a single ordinary differential equation for the real function $Z$, where the right-hand side is the known fifth-order polynomial in $Z$, namely

$$
\left(\frac{\mathrm{d} Z}{\mathrm{~d} t}\right)^{2}=\mathcal{P}_{5}(Z)
$$

where

$$
\begin{aligned}
\mathcal{P}_{5}(Z)= & -\left(P W Z^{2}+N Z-Q\right)^{2} \\
+ & \left(6 W Z+\left|a_{0}\right|^{2}\right)^{3} \times \\
& \times\left(-2 W Z+\left|b_{0}\right|^{2}\right)\left(-2 W Z+\left|c_{0}\right|^{2}\right),
\end{aligned}
$$

and the notation has been introduced

$$
\begin{aligned}
P & =3 P_{a}-P_{b}-P_{c} \\
N & =P_{a}\left|a_{0}\right|^{2}+P_{b}\left|b_{0}\right|^{2}+P_{c}\left|c_{0}\right|^{2}+3 \omega_{a}-\omega_{b}-\omega_{c} \\
P_{a} & =3 V_{a a a a}-2 V_{a b a b}-2 V_{a c a c} \\
P_{b} & =6 V_{a b a b}-V_{b b b b}-2 V_{b c b c} \\
P_{c} & =6 V_{a c a c}-2 V_{b c b c}-V_{c c c c} \\
Q & =\operatorname{Re}\left(a_{0}^{* 3} b_{0} c_{0}\right)
\end{aligned}
$$

The solution of (A5) has the form

$t=\int_{0}^{Z} \frac{\mathrm{d} Z}{\sqrt{\mathcal{P}_{5}(Z)}}$

where the integration is performed between two neighbouring roots of the polynomial with the opposite signs
(Shemer and Stiassnie, 1985). Obviously, $Z$ is periodic in $t$, with the period

$T=2 \int_{Z_{L}}^{Z_{R}} \frac{\mathrm{d} Z}{\sqrt{\mathcal{P}_{5}(Z)}}$

where $Z_{L}$ and $Z_{R}$ are the neighbouring roots of (A6), such that $Z_{L}<0<Z_{R}$.

The explicit solution to Eq. (A5), in principle, could be written out in terms of hyperelliptic functions. However, we are interested in the class of initial conditions when the initial amplitude of the sidebands is much smaller than that of the fundamental

$\left|b_{0}\right|^{2} \simeq\left|c_{0}\right|^{2} \ll\left|a_{0}\right|^{2}$,

and, without the loss of generality, $\operatorname{Im}\left(a_{0}\right) \equiv 0$.

Then, using $\varepsilon=O\left(b_{0}^{2}, c_{0}^{2}\right)$ as a small parameter, we can rewrite Eq. (A5) in the form

$$
\begin{gathered}
\left(\frac{\mathrm{d} Z}{\mathrm{~d} t}\right)^{2}=-\left(P W Z^{2}+N Z-\varepsilon \beta\left|a_{0}\right|^{3}\right)^{2} \\
+\left(\left|a_{0}\right|^{2}+6 W Z\right)^{3}(\varepsilon-2 W Z)^{2},
\end{gathered}
$$

where $\beta=\operatorname{Re}\left(b_{0} c_{0}\right) /\left|b_{0}\right|^{2}$ measures the initial phase of the satellites (in particular, $\beta=0$ if $\operatorname{Ph}\left(b_{0}\right)=\operatorname{Ph}\left(c_{0}\right)=$ $\pm \pi / 4$ ).

Since $\varepsilon$ is small, roots of the polynomial in the righthand side of Eq. (A11) have different orders, at least two roots being much smaller then the other ones. This prompts to look for the roots as

$$
\begin{aligned}
& r_{1}=r_{1}^{(0)}+O(\varepsilon), r_{2}=r_{2}^{(0)}+O(\varepsilon), r_{3}=r_{3}^{(0)}+O(\varepsilon), \\
& r_{4}=\varepsilon r_{4}^{(1)}+O\left(\varepsilon^{2}\right), r_{5}=\varepsilon r_{5}^{(1)}+O\left(\varepsilon^{2}\right) .
\end{aligned}
$$

It is easy to see that the smaller roots $r_{4}, r_{5}$, to a leading order, are

$r_{4}=\frac{\left|a_{0}\right|^{3}(\beta+1)}{N+2\left|a_{0}\right|^{3} W}, \quad r_{5}=\frac{\left|a_{0}\right|^{3}(\beta-1)}{N-2\left|a_{0}\right|^{3} W}$. 

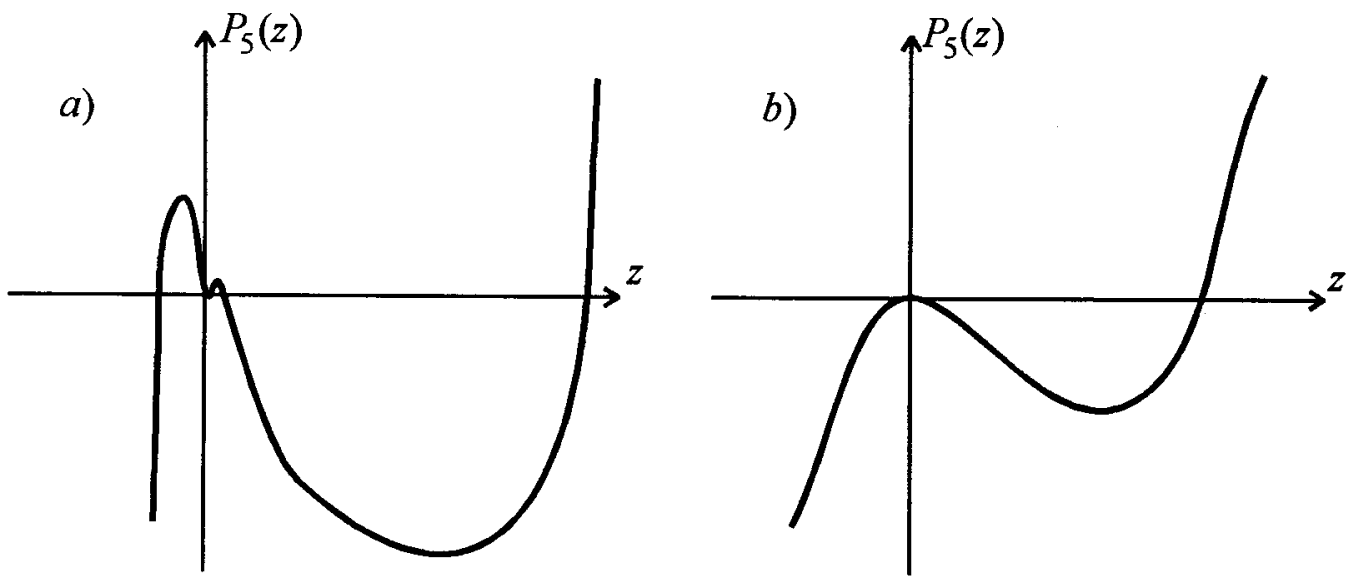

Fig. A2. Typical form of the potential $\mathcal{P}_{5}(a)$ within and (b) outside of the instability domain.

Expressions (A13) are valid, to a leading order, for all parameter values except for the neighbourhood of the points where $N \pm 2\left|a_{0}\right|^{3} W=0$.

The larger roots $r_{1}, r_{2}, r_{3}$, to a leading order, are obtained from the cubic equation

$4 W^{2}\left(6 W r+\left|a_{0}\right|^{2}\right)^{3}-(P W r+N)^{2}=0$.

An interesting special case occurs when

$\left[972\left(P\left|a_{0}\right|^{2}-6 N\right) W^{2}-P^{3}\right]\left(P\left|a_{0}\right|^{2}-6 N\right)=0,(A 1$

and two roots of Eq. (A14) coalesce. In particular, if the condition

$$
P\left|a_{0}\right|^{2}-6 N=0
$$

is satisfied, then

$$
r_{1,2}=-\frac{\left|a_{0}\right|^{2}}{24 W}-\frac{3}{4} \frac{N}{P W}, \quad r_{3}=\frac{1}{864} \frac{P^{2}}{W^{3}}-\frac{N}{P W} .
$$

In another case, if

$$
972\left(P\left|a_{0}\right|^{2}-6 N\right) W^{2}-P^{3}=0,
$$

then

$$
r_{1,2}=\frac{\left|a_{0}\right|^{2}}{3 W}-\frac{3 N}{P W}, \quad r_{3}=-\frac{\left|a_{0}\right|^{2}}{24 W}-\frac{3}{4} \frac{N}{P W} .
$$

Roots of $\mathcal{P}_{5}(Z)$ for the case $p=0$ are plotted in Fig. A1 as functions of $q$. Due to large difference of the orders of the roots, the plot is schematic and is intended to demonstrate just the basic features of the structure. Two examples of the form of the potential $\mathcal{P}_{5}(Z)$ are shown if Fig. A2.

Now we recall that in the solution (A8) the upper integration limit is allowed to change between two real roots of opposite signs with the smallest absolute value. It is clear, however, that the roots $r_{4}$ and $r_{5}$ have the smallest absolute values and opposite signs if $N<-2\left|a_{0}\right|^{3} W$ or $N>2\left|a_{0}\right|^{3} W$. This means that the amplitude of modulations is very small there.
Instability is possible only if

$$
-2\left|a_{0}\right|^{3} W<N<2\left|a_{0}\right|^{3} W
$$

then both $r_{4}$ and $r_{5}$ are positive, and $Z$ is allowed to vary between the smallest of $\left(r_{4}, r_{5}\right)$, that is,

$$
r_{+}=\varepsilon \frac{\left|a_{0}\right|^{3}(\beta+1)}{N+2\left|a_{0}\right|^{3} W}
$$

and the negative root of the polynomial $\mathcal{P}_{5}(Z)$, which can be, with good accuracy, estimated as

$$
r_{-}=-\frac{\left|a_{0}\right|^{2}}{24 W}-\frac{3}{4} \frac{N}{P W} .
$$

This defines the amplitude of the modulations. The amplitude reaches its maximum while approaching the neighbourhood of the point $N=2\left|a_{0}\right|^{3} W$; at this point, the asymptotic expansion breaks.

Region in the parameter space where inequality (A16) holds can be termed the domain of instability. Within it the solution can be written out, to the leading order, as

$$
\begin{aligned}
t= & \int_{0}^{Z} \frac{\mathrm{d} r}{(r-O(\varepsilon))} \times \\
& \times \frac{1}{\sqrt{4 W^{2}\left(6 W r+\left|a_{0}\right|^{2}\right)^{3}-(P W r+N)^{2}}}
\end{aligned}
$$

where $Z$ is allowed to change between 0 and $r_{-}$.

To the leading order, the period, defined by (A9), is infinite. More realistic values for the period can be obtained by the extension of the asymptotic procedure to higher orders. Note, however, that if $N=0$, then the small roots coincide, and the solution has infinite period in all orders. In this case, there is infinite time between modulations of the three-wave system governed by (A1), existence of this regime being easily verified numerically.

Thus, the approximate expression (A17) for the exact hyperelliptic solution to (A1) within the instability domain (A16) is formulated in the form of an elliptic 
integral. If, however, condition (A15) is satisfied somewhere within this domain, two more roots of the polynomial coincide. In this case, the solution can be further simplified to trigonometric form.

Acknowledgements. We are grateful to Dr. Guillemette Caulliez for numerous important discussions of three-dimensional windwave patterns. The work was supported by the US Office of Naval Research (Grant 00014-94-1-0532) and Russian Foundation for Basic Research (Grant No. 98-05-64714).

\section{References}

Annenkov, S. Yu., and Shrira, V. I., New numerical method for surface waves hydrodynamics based on the Zakharov equation, J. Fluid Mech., Submitted.

Badulin, S. I., and Shrira, V. I., Global dynamics in simple models of three-dimensional water wave patterns, Eur. J. Mech., Submitted.

Bowman, C., and Newell, A. C., Natural patterns and wavelets, Rev. Mod. Phys., 70, 289-301, 1998.

Collard F., and Caulliez G., Oscillating crescent-shaped water wave patterns, Phys. Fluids, Submitted.

Craik, A. D. D., Wave Interactions and Fluid Flows, Cambridge University Press, 1985.

Crawford, D. R., Saffman, P. G., and Yuen, H. C., Evolution of a random inhomogeneous field of nonlinear deep-water gravity waves, Wave Motion, 2, 1-16, 1980.

Kartashova, E. A., On properties of weakly nonlinear wave interactions in resonators, Physica $D, 54,125-134,1991$.

Krasitskii, V. P., On reduced Hamiltonian equations in the nonlinear theory of water surface waves, J. Fluid Mech., 272, 1-20, 1994.

Krasitskii, V. P., and Kalmykov, V. A. Four-wave reduced equa- tions for surface gravity waves, Izvestiya, Atmospheric and Oceanic Physics, 29, 222-228, 1993.

Kusaba, T., and Mitsuyasu, H., Nonlinear instability and evolution of steep water waves under wind action, Rep. Res. Inst. Appl. Mech. Kyushu University, 33, No. 101, 33-64, 1986.

McLean, J. W., Instabilities of finite-amplitude water waves, J. Fluid Mech., 114, 315-330, 1982.

Melville, W. K., The instability and breaking of deep-water waves, J. Fluid Mech., 115, 165-185, 1982.

Shemer, L., and Stiassnie, M., Initial instability and long-time evolution of Stokes waves. In: Y.Toba and H. Mitsuyasu (eds.), The Ocean Surface, p. 51-57, D. Reidel Publishing Company, 1985.

Shrira, V. I., Badulin, S. I., and Kharif, C., A model of water wave 'horse-shoe' patterns. J. Fluid Mech., 318, 375-404, 1996.

Shrira, V. I., Badulin, S. I., and Voronovich, A. G., EM wave scattering by 3 -d wind wave patterns at grazing angles, in preparation.

Stiassnie, M., and Shemer, L., Energy computations for evolution of class I and class II instabilities of Stokes waves, J. Fluid Mech., 174, 299-312, 1987.

Su, M.-Y., Three-dimensional deep-water waves. Part I. Experimental measurements of skew and symmetric wave patterns, J. Fluid Mech., 124, 73-108, 1982.

Su, M.-Y., Bergin, M., Marler, P., and Myrick, R., Experiments on non-linear instabilities and evolution of steep gravity wave trains, J. Fluid Mech., 124, 45-72, 1982.

Su, M.-Y., and Green, A. W., Coupled two- and three-dimensional instabilities of surface gravity waves, Phys. Fluids, 27, 2595$2597,1984$.

Yuen, H. C., and Lake, B. M., Nonlinear dynamics of deep-water gravity waves, Adv. Appl. Mech., 22, 67-229, 1982.

Zakharov, V. E., Stability of periodic waves of finite amplitude on the surface of a deep fluid, J. Appl. Mech. Tech. Phys. (USSR), $9,86-94,1968$. 